\title{
Raman Spectroscopy: Guiding Light for the Extracellular Matrix
}

\section{OPEN ACCESS}

Edited by:

Francesca Taraballi, Houston Methodist Research Institute, United States

Reviewed by: Antonino Natalello, University of Milano Bicocca, Italy

Cristian Pablo Pennisi, Aalborg University, Denmark

*Correspondence: Mads S. Bergholt mads.bergholt@kcl.ac.uk

Michael B. Albro albro@bu.edu

$$
\begin{array}{r}
\text { Specialty section: } \\
\text { This article was submitted to } \\
\text { Biomaterials, } \\
\text { a section of the journal } \\
\text { Frontiers in Bioengineering and } \\
\text { Biotechnology }
\end{array}
$$

Received: 20 August 2019 Accepted: 16 October 2019 Published: 01 November 2019

Citation:

Bergholt MS, Serio $A$ and Albro MB (2019) Raman Spectroscopy: Guiding Light for the Extracellular Matrix.

Front. Bioeng. Biotechnol. 7:303. doi: 10.3389/fbioe.2019.00303

\author{
Mads S. Bergholt ${ }^{1 *}$, Andrea Serio ${ }^{1}$ and Michael B. Albro ${ }^{2 *}$ \\ ${ }^{1}$ Centre for Craniofacial and Regenerative Biology, King's College London, London, United Kingdom, ${ }^{2}$ Department of \\ Mechanical Engineering, Boston University, Boston, MA, United States
}

The extracellular matrix (ECM) consists of a complex mesh of proteins, glycoproteins, and glycosaminoglycans, and is essential for maintaining the integrity and function of biological tissues. Imaging and biomolecular characterization of the ECM is critical for understanding disease onset and for the development of novel, disease-modifying therapeutics. Recently, there has been a growing interest in the use of Raman spectroscopy to characterize the ECM. Raman spectroscopy is a label-free vibrational technique that offers unique insights into the structure and composition of tissues and cells at the molecular level. This technique can be applied across a broad range of ECM imaging applications, which encompass in vitro, ex vivo, and in vivo analysis. State-of-the-art confocal Raman microscopy imaging now enables label-free assessments of the ECM structure and composition in tissue sections with a remarkably high degree of biomolecular specificity. Further, novel fiber-optic instrumentation has opened up for clinical in vivo ECM diagnostic measurements across a range of tissue systems. A palette of advanced computational methods based on multivariate statistics, spectral unmixing, and machine learning can be applied to Raman data, allowing for the extraction of specific biochemical information of the ECM. Here, we review Raman spectroscopy techniques for ECM characterizations over a variety of exciting applications and tissue systems, including native tissue assessments (bone, cartilage, cardiovascular), regenerative medicine quality assessments, and diagnostics of disease states. We further discuss the challenges in the widespread adoption of Raman spectroscopy in biomedicine. The results of the latest discovery-driven Raman studies are summarized, illustrating the current and potential future applications of Raman spectroscopy in biomedicine.

\footnotetext{
Keywords: label-free imaging, Raman spectroscopy, extracellular matrix, collagen, glycosaminoglycans, fiberoptic diagnostics, tissue engineering
}

\section{INTRODUCTION}

\section{The Extracellular Matrix}

The extracellular matrix (ECM) is a fundamental component of all biological tissues. The ECM consists of complex, integrated networks of extracellular biomolecules that serve to provide tissues with structural and biochemical support. ECM structures give rise to a multitude of tissue-specific functionalities, providing mechanical stability, molecular transport barriers, junctions for cell adhesion, transduction of mechanical cues, and reservoirs of growth factors. The ECM is composed of a myriad of biomolecular constituents, including fibrous proteins (e.g., collagen, elastin), glycoproteins (e.g., laminin, fibrillin, fibronectin), glycosaminoglycans (GAG) 
(e.g., chondroitin-sulfate, heparin, hyaluronan), GAG-rich proteoglycans (e.g., aggrecan, versican, decorin), and lipids (e.g., cholesterol, phospholipids). These constituents are organized in unique, tissue-specific arrangements that are optimized in terms of content, distribution, and alignment to achieve requisite structure-function relationships. For instance, musculoskeletal connective tissues consist of collagen networks interspersed with dense proteoglycans, giving rise to requisite combinations of tensile and compressive mechanical resistance (Chahine et al., 2004) and low friction interfaces (Krishnan et al., 2003). In the cardiovascular system, the arterial wall is composed of an interwoven network of elastic and collagenous fibers, providing vessels with an important combination of pulsatile energy efficiency and rupture strength (Cocciolone et al., 2018). In the nervous system, lipid-rich myelin sheaths surround and insulate neurons, allowing for effective long-range transmission of electrical impulses. Ultimately, the make-up and organization of the ECM is vital for the functionality, health, and longevity of biological tissues. Furthermore, pathological tissue disorders are typically associated with the disruption of or abnormalities to the ECM (Bonnans et al., 2014). Prominent examples include: (i) fibrosis from aberrant matrix protein secretion, as characterized in pathology of vital organs [e.g., lung (White, 2015), kidney (Duffield, 2014), heart (Prabhu and Frangogiannis, 2016), and liver (Kisseleva, 2017)], (ii) musculoskeletal tissue degeneration from the break-down of the load-supporting ECM (Krishnan and Grodzinsky, 2018), and (iii) metastatic cancers from the invasion of malignant cells through ECM barriers (Gilkes et al., 2014). Accordingly, the ability to characterize the make-up and organization of the ECM is critical for understanding the onset of pathologies and for developing novel disease-modifying therapeutics.

\section{Imaging of the Extracellular Matrix}

Imaging techniques, such as conventional microscopy, have long served as tools for studying the ECM. Today, characterizations of the ECM continue to rely heavily on conventional histology staining techniques, such as colorimetric dyes for general ECM constituents (e.g., Alcian Blue and safranon O for GAG, Picrosirius Red for collagen) and colorimetric or fluorescentconjugated antibodies for imaging specific molecular ECM constituents. While these methods offer detailed and specific characterizations of protein distributions, they are associated with a host of well-documented limitations (Hyllested et al., 2002), such as sample fixation artifacts (Pousty et al., 1975; Wilson and Gardner, 1980), non-stoichiometric dye binding (Puchtler et al., 1988), and lack of dye binding specificity (Scott and Stockwell, 1967; Jubb and Eggert, 1981), which limit their ability to provide reliable quantitative or even semi-quantitative assessments of tissue ECM distributions. Further, histological staining is limited by its association with highly laborious sample preparation protocols. To complement and potentially advance beyond conventional staining techniques, microscopy is continually evolving, enabling novel imaging capabilities from the tissue-scale to nano-scale level.

A palette of imaging technologies has been used for characterizations of the ECM, including widefield fluorescence microscopy, confocal microscopy, polarized light microscopy (PLM), and super-resolution microscopy (Brightman et al., 2000). Moreover, a number of advanced, non-linear, labelfree imaging modalities for studying the ECM and cell-ECM interactions have been developed, including second harmonic generation (SHG) microscopy (Burke et al., 2013), two-photon excited fluorescence (TPEF) microscopy and terahertz (THz) spectroscopy imaging (Jung et al., 2012a,b; Stantchev et al., 2018). These imaging techniques provide the advantage of offering detailed insights into the structural characteristics of tissue ECMs. Further, ultrastructural imaging can be archived using scanning electron microscopy (SEM) (Santi et al., 2016) or atomic force microscopy (AFM) (Jorba et al., 2017), while specific compositional imaging can be achieved with complementary mass spectrometry (Gessel et al., 2015). A range of optical spectroscopy techniques, such as Fourier transform infrared (FT-IR) spectroscopy (Cheheltani et al., 2014), have also been extensively applied to characterize the ECM. FT-IR measures the vibrational absorption spectrum of molecules and has been used to elucidate the distribution of collagen in sectioned tissues (Spalazzi et al., 2013). State-of-the-art FT-IR microscopic imaging using focal plane array (FPA) detectors offers rapid snapshot spectral imaging for biomolecular analysis. FT-IR, however, exhibits several limitations, such as relatively low imaging resolution and incompatibility with imaging aqueous ECM tissue environments.

Raman spectroscopy is an inelastic light scattering technique that offers a unique potential for advanced ECM characterizations. Raman scattering occurs when light induces a change in the polarizability of molecules. In recent years, the use of Raman spectroscopy in biomedical applications has received growing attention due to its ability to achieve robust quantitative assessments of the biochemical composition of biological tissues. Raman spectral peaks are highly specific to molecular chemistry (Shetty et al., 2006) with intensities that are proportional to molecular content (Bergholt et al., 2017), allowing for critical assessments of both the ratios and concentrations of individual ECM constituents in a specimen. Further, Raman spectral acquisitions do not require molecular labeling or contrast agents, allowing for non-invasive or minimally-invasive ECM assessments. Two rapidly expanding applications for Raman spectroscopy in biomedicine are: i) Raman spectroscopic imaging (Raman microscopy) for characterizing the makeup and spatial distribution of ECM constituents throughout biological tissue sections, and ii) fiber-optic based Raman spectroscopy for diagnostics of ECM alterations in the in vivo environment. For microscopy-based applications, Raman spectroscopy is compatible with hydrated tissues and can yield images with diffraction-limited spatial resolution, allowing for the generation of high resolution quantitative images of the ECM distribution in live or unprocessed tissue specimens. Fiber-optic based diagnostics benefit considerably from the label-free nature of Raman acquisitions, allowing for minimally invasive quantifications of critical ECM alterations that are associated with disease states. Overall, Raman spectroscopy is now widely applicable for an extensive range of in vitro, ex vivo, and in vivo ECM-related characterizations and diagnostics. 
These developments have occurred alongside the establishment of advanced computational methods, including multivariate algorithms, spectral unmixing, and machine learning approaches in order to extract and characterize the ECM tissue structure and composition at the molecular level. These computational methods have greatly aided the development of Raman spectroscopy ECM characterizations in the areas of imaging and diagnostics.

In this article, we review the role and applications of state-of-the-art Raman spectroscopy for ECM characterizations. The results of the latest Raman microscopy imaging and fiber-optic diagnostic techniques are summarized, spanning from regenerative medicine assessments to disease diagnostics, and illustrating both current and potential future applications in biomedicine.

\section{RAMAN SPECTROSCOPY}

Conventional (spontaneous) Raman scattering is an inelastic interaction between light and molecules. When light interacts with a molecule, it can be excited to a short-lived virtual state that immediately falls back to a vibrational excited state in the electronic ground state (Figure 1A). Because of this interaction, a small amount of energy is transferred or removed from the molecule and the resulting scattered light is red shifted (stokes) or blue shifted (anti-stokes) containing encoded vibrational molecular information (i.e., fingerprints). For this reason, Raman scattering of tissues offers a wealth of information about the vibrational structure of their compositional proteins, GAGs, lipids, and DNA. Raman spectra are often recorded in the so-called fingerprint region $\left(400-1,800 \mathrm{~cm}^{-1}\right)$ that contains relatively weak but highly specific Raman peaks, allowing for ECM assessments with a remarkably high degree of biomolecular specificity. Recently, additional attention has been drawn to the use of the high wavenumber region $\left(2,800-3,600 \mathrm{~cm}^{-1}\right)$, which contains Raman bands that are less specific but exhibit a higher degree of signal intensity.

\section{Raman Spectroscopy Instrumentation}

Raman spectra of tissues can be measured using a microscope or custom fiber-optics. A state-of-the-art confocal Raman microscope is shown in Figure 1B. Briefly, the laser is coupled into the microscope using a single-mode fiber and illuminated onto the sample with a microscope objective. Raman spectroscopic-based confocal imaging can be achieved by collecting the backscattered light using a fiber. The single fiber acts as pinhole and couples the light into a high-throughput spectrometer that disperses it onto a charge coupled device (CCD) camera. A valuable growing application of Raman microscopic imaging is the generation of hyperspectral Raman images, whereby spectra are acquired at discrete positions over the surface or exposed cross-section of a specimen and analysis is performed to generate a spectral-based image. For these applications, rapid raster scanning is typically performed using a motorized or piezoelectric stage. Despite the traditional shortcoming of weak intensity tissue signals, rapid advances in instrumentation and detectors have improved the speed at which Raman spectral images can be measured, reducing the acquisition time of high resolution images to only hours or minutes. Several variants of Raman microscopy systems exist, such as confocal Raman microscopy (Puppels et al., 1990), light sheet Raman microscopy (Oshima et al., 2012), and line scan Raman microscopy (Grauw et al., 1997). In general, fiberbased confocal microscopy setups offer superior imaging of the ECM compared to conventional non-confocal setups, in terms of Raman signal quality. This can be attributed to the rejection of out-of-focus scattered light. Integration times for measuring Raman images of tissues can be as low as $0.1 \mathrm{~s}$ for a $532 \mathrm{~nm}$ high numerical aperture (NA) confocal Raman setup. Hence, a full hyperspectral image (e.g., $100 \times 100$ pixels) can be measured within $\sim 20$ minutes. Single Raman spectra of tissues can also be measured using fiber-optic sampling. Figure $\mathbf{1 C}$ shows an example of an endoscopic compatible Raman platform used for the in vivo tissue analysis of colon cancer. The laser light is coupled into a $2 \mathrm{~mm}$ bifurcated fiber-optic probe that delivers the laser and collects the Raman scattered light within $1 \mathrm{~s}$, which can then be analyzed using computational methods in real-time.

The choice of laser excitation wavelength is one of the most important parameters when performing Raman spectroscopy. Generally, wavelengths are selected based on a compromise between reducing background autofluorescence and maximizing Raman signal intensities, both of which increase with decreasing wavelength (the Raman signal scales with the 4th power of the excitation frequency). The optimization of this balance is highly dependent on the sample type and application. Most tissues will give sufficient Raman signal and desirable low autofluorescence under near-infrared (NIR) 785-1,048 nm laser excitation (Lim et al., 2011; You et al., 2017). There are exceptions, as some tissues can exhibit prohibitively high autofluorescence even under NIR excitation [e.g., liver, spleen, heart, kidneys, lung tissue (Huang et al., 2011)]. For samples with particularly low degrees of autofluorescence (e.g., cartilage, bone, cell monolayers), it is often advantageous to use shorter excitation wavelengths, such as 633 or $532 \mathrm{~nm}$, which significantly speeds up the acquisition time due to the generation of stronger signals. It is important to consider that Raman spectra obtained with different laser excitation wavelengths should, in general, not be compared directly due to possible molecular resonance effects.

The imaging resolution of Raman microscopy depends on the specific type of system but is ideally diffraction-limited and, therefore, depends heavily on the excitation wavelength and choice of objective. For a state-of-the-art confocal Raman system with visible excitation, the lateral resolution can reach as low as $300 \mathrm{~nm}$ and the depth resolution can reach less than $500 \mathrm{~nm}$ for sufficiently small pinhole settings.

\section{Raman Microscopy Sample Preparation}

For Raman microscopy-based applications, the compatibility of sample processing techniques must always be considered. A major advantage of Raman spectroscopy is its exceptional compatibility with aqueous environments. As such, imaging can be performed directly on hydrated tissues in buffered saline solution without the implementation of any sample processing (Bergholt et al., 2016a) (e.g., fixation, dehydration, or 
A
Raman scattering

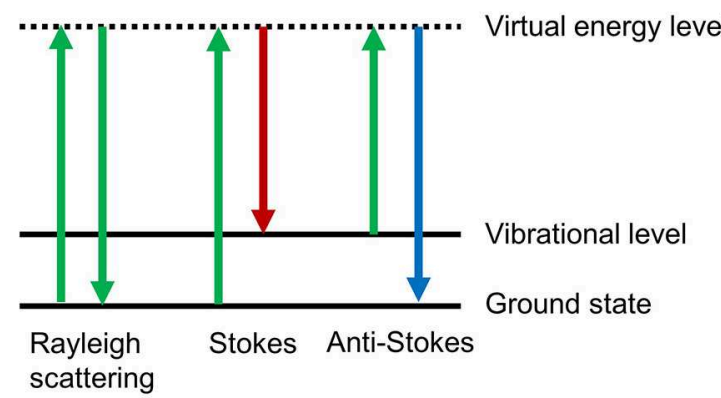

B Confocal Raman microscope

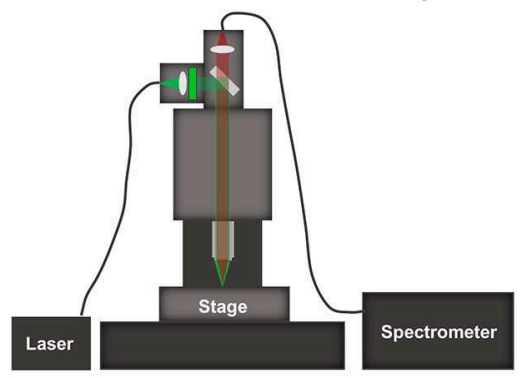

C
Fiber-optic Raman system

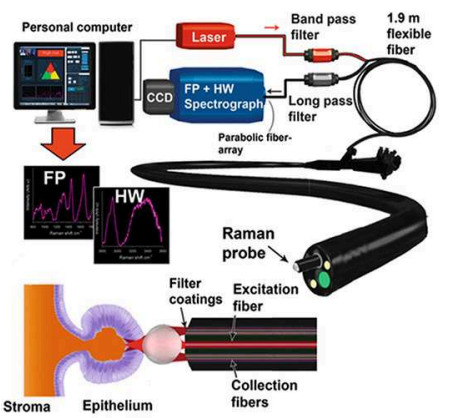

FIGURE 1 | (A) The Raman effect. (B) Schematic of confocal Raman microscopy platform for imaging. (C) Example of fiber-optic Raman spectroscopy for endoscopy measurements in the gastrointestinal tract [Reprinted with permission from Bergholt et al. (2016b)].

embedding). For the acquisition of large hyperspectral images, it is often advantageous to analyze specimens with flat surfaces in order to maintain a consistent focus across a large region of interest. Sample flattening can be readily achieved with cryostat trimming or, if the preservation of tissue viability is required, with a vibratome or a template cutting block. Tissues can also be sectioned through conventional histology sectioning protocols (Bergholt et al., 2018). One drawback of Raman imaging is that paraffin embedding is not suitable for Raman acquisitions due to significant spectral interference. Both digital and experimental deparaffinization approaches have been explored (Tfayli et al., 2009; Gaifulina et al., 2016). Tissues can be readily imaged in either a fresh or formalin fixed state, though formalin fixation introduces subtle differences in the Raman spectra due to molecular crosslinking (Huang et al., 2003a; Meade et al., 2010). Further, it is important to note that the use of conventional microscopy glass slides is not suitable for non-confocal Raman spectroscopy and typically requires the use of special substrates [e.g., aluminum, steel, calcium fluoride $\left(\mathrm{CaF}_{2}\right)$ or magnesium fluoride $\left.\left(\mathrm{MgF}_{2}\right)\right]$ that induce negligible background signal. Stateof-the-art confocal Raman microscopy with high magnification objectives and a small pinhole, however, offers imaging with essentially no contribution from conventional glass substrate even for imaging of a monolayer of cells (Kallepitis et al., 2017).

\section{Raman Spectroscopy Analysis}

Due to the large amount of data obtained from hyperspectral Raman images, computational analysis is essential in order to extract molecular information. Before Raman spectra are analyzed, they must be preprocessed in order to reduce confounding factors, such as background autofluorescence and noise. Common methods to do so include cosmic ray rejection, autofluorescence subtraction, dark signal subtraction, and normalization (Lieber and Mahadevan-Jansen, 2003; Zhao et al., 2007; Hedegaard et al., 2016). Further, to improve reproducibility across instruments, standardization of the Raman intensities should, in general, be performed through system response correction using a NIST standards, such as standard reference materials range (e.g., SRM2241 for $785 \mathrm{~nm}$ laser excitation). For sufficiently flat samples, semi-quantitative images may be derived from the absolute signal, whereas spectral normalization schemes may be required to achieve artifactfree images for samples with thickness variations. In temporal studies, it is often important to combine Raman images from several different time points in order to perform quantitative image analysis. Due to varying focus of the microscope objective and, therefore, varying absolute intensities, this requires careful standardization of the Raman images, such as through spectral normalization (Hedegaard et al., 2016; Kallepitis et al., 2017).

There has been remarkable advancement in the development of computational methods for analysis. Overall, there exist several different approaches to performing spectral analysis of Raman images. The simplest approach is univariate peak analysis by mapping the intensities of a single Raman peak, gaussian peak fitting, or peak intensity ratios. A comprehensive list of tentatively assigned Raman peaks can be found in Table 1. While univariate peak imaging can offer approximations of the visualization of highly concentrated ECM components (Bergholt 
TABLE 1 | Tentative assignments of Raman peaks of the extracellular matrix and cells.

\begin{tabular}{|c|c|c|c|}
\hline Raman peak & $\begin{array}{l}\text { Tentative } \\
\text { assignment }\end{array}$ & $\begin{array}{l}\text { Associated } \\
\text { molecule }\end{array}$ & References \\
\hline 785-795 & $v_{\mathrm{S}}(\mathrm{O}-\mathrm{P}-\mathrm{O})$ & DNA & Kuhar et al., 2018 \\
\hline 820-939 & $v(\mathrm{C}-\mathrm{C})$ & $\begin{array}{l}\text { Proteins } \\
\text { (collagen) }\end{array}$ & Janko et al., 2010 \\
\hline 960 & $v\left(\mathrm{PO}_{4}\right)$ & Hydroxyapatite & $\begin{array}{l}\text { Bergholt et al., } \\
2016 a\end{array}$ \\
\hline 1,002-1,004 & $v_{s}(\mathrm{C}-\mathrm{C})$ & $\begin{array}{l}\text { Phenylalanine } \\
\text { of proteins }\end{array}$ & Lim et al., 2011 \\
\hline 1,063 & $v_{s}(S=0)$ & GAG & $\begin{array}{l}\text { Demers et al., } \\
2015\end{array}$ \\
\hline 1,080 & $v(\mathrm{C}-\mathrm{C})$ & Lipids & $\begin{array}{l}\text { Czamara et al., } \\
2015\end{array}$ \\
\hline $1,230-1,280$ & Amide III & Proteins & Rygula et al., 2013 \\
\hline $1,375-1,410$ & $v_{\mathrm{s}}\left(\mathrm{COO}^{-}\right)$ & GAGs & $\begin{array}{l}\text { Bergholt et al., } \\
2016 a\end{array}$ \\
\hline $1,400-1,500$ & $\begin{array}{l}\mathrm{b}\left(\mathrm{CH}_{2} / \mathrm{CH}_{3}\right), \\
\mathrm{sc}\left(\mathrm{CH}_{2} / \mathrm{CH}_{3}\right), \\
\mathrm{b}\left(\mathrm{CH}_{2}\right)\end{array}$ & Lipids & Kuhar et al., 2018 \\
\hline $1,445-1,456$ & $\begin{array}{l}\mathrm{b}\left(\mathrm{CH}_{2} / \mathrm{CH}_{3}\right) \\
\mathrm{sc}\left(\mathrm{CH}_{2} / \mathrm{CH}_{3}\right) \\
\mathrm{b}\left(\mathrm{CH}_{2}\right)\end{array}$ & Proteins & $\begin{array}{l}\text { Lim et al., 2011; } \\
\text { Esmonde-White, } \\
2014\end{array}$ \\
\hline 1,650-1,680 & Amide I $v(C=C)$ & Proteins & Rygula et al., 2013 \\
\hline 1,745 & $v(C=0)$ & Lipids & $\begin{array}{l}\text { Movasaghi et al., } \\
2007\end{array}$ \\
\hline $2,850-2,854$ & $v_{\mathrm{s}}\left(\mathrm{CH}_{2}\right)$ & Lipids & $\begin{array}{l}\text { Czamara et al., } \\
2015 \text {; Lu et al., } \\
2015\end{array}$ \\
\hline 2,880 & $v_{\text {as }}\left(\mathrm{CH}_{3}\right)$ & Lipids & $\begin{array}{l}\text { Mo et al., 2009; } \\
\text { Czamara et al., } \\
2015\end{array}$ \\
\hline $2,931-2,940$ & $v\left(\mathrm{CH}_{3}\right)$ & Proteins & Mo et al., 2009 \\
\hline $3,100-3,600$ & $v(\mathrm{OH})$ & $\mathrm{H}_{2} \mathrm{O}$ & $\begin{array}{l}\text { Leikin et al., 1997; } \\
\text { Lu et al., } 2015\end{array}$ \\
\hline
\end{tabular}

The Raman spectra of various tissue biomolecules are highly overlapping, and the peak position is often reported with variability of $\sim \pm 1-5 \mathrm{~cm}^{-1}$ due to instrument variability.

et al., 2016a), it often yields limited molecular specificity due to the inherent overlapping spectra of individual ECM constituents; Figure 2 shows an example of spectra of purified collagen, GAG, and water, as well as the compound spectrum from native articular cartilage tissue. Alternatively, the implementation of more advanced multivariate or machine learning approaches can be used to extract accurate quantitative information for specific ECM constituents. Multivariate and machine learning offer, in general, more accurate quantitative analysis, as they consider the full range of spectral information. These techniques generally come in two forms: unsupervised and supervised analysis.

\section{Unsupervised Algorithms Principal Component Analysis}

The most commonly used technique is principal component analysis (PCA) (Shetty et al., 2006). PCA finds the orthogonal principal components (PCs) of a dataset that successively account for the largest variability in the dataset. For instance, PCA can be

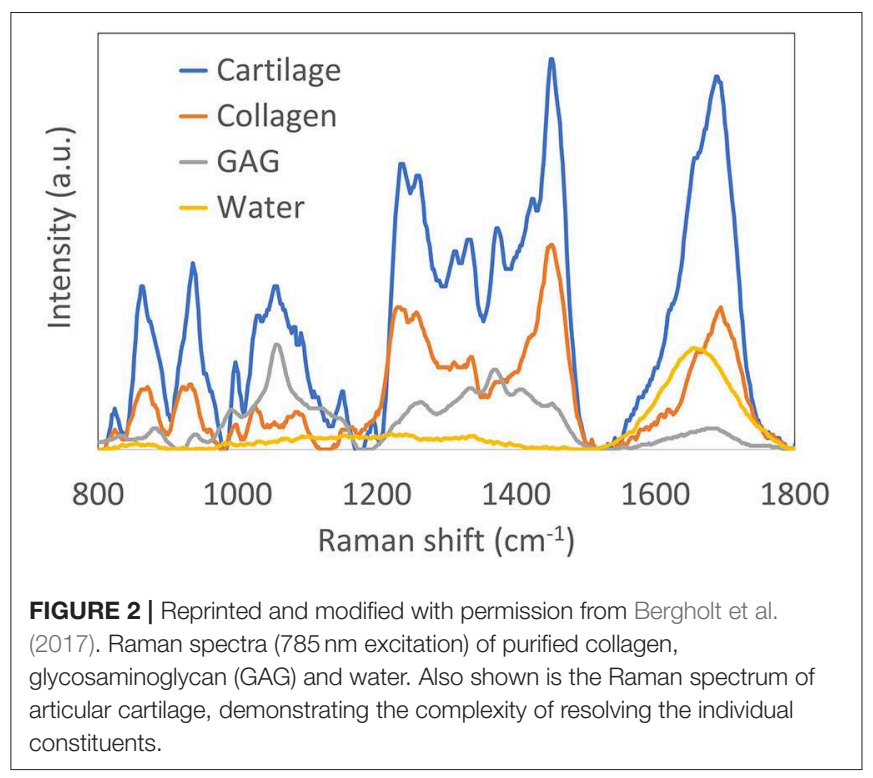

used to reduce the dimension of the spectral data significantly while reducing noise and providing more robust models. In general, PCA can be applied as an exploratory technique for hyperspectral Raman imaging, as it enables easy identification of the largest spectral variability, as well as imaging artifacts. PCA is often combined with linear discriminant analysis (LDA) in supervised settings. A major limitation of PCA is that it does not offer pure estimates of components.

\section{Multivariate Curve Resolution (MCR)}

This powerful technique is applied to deconvolve a specimen's Raman spectra into the underlying spectra of its dominant ECM constituents through variance in the data (Albro et al., 2018). In contrast to PCA, MCR offers estimates of the concentrations of ECM constituents in a tissue. As a limitation, since the deconvolution analysis is generally ill-determined, MCR can encounter difficulties for the analysis of complex tissues with an excessive number of concentrated ECM components or for experiments with insufficient sized data sets. A good practice to validate the modeling capability of MCR is, therefore, to correlate the MCR components with Raman spectra of purified or commercially available components.

\section{Clustering Analysis}

Another type of unsupervised analysis is clustering techniques, such as $k$-means or nearest neighbor clustering. In $\mathrm{k}$-means clustering the spectra are partitioned into $k$ clusters where each spectrum belongs to the cluster with the nearest mean. These exploratory techniques can be used to group similar spectra and can give insights into tissues that have similar or different composition (Kong et al., 2013).

\section{Supervised Algorithms \\ Partial Least Squares (PLS)}

Partial least squares (PLS) represents a regression technique like PCA, but the latent variables (LVs) are further rotated 
to correlate with reference concentrations. This technique has few applications for Raman imaging, but is better suited for spectroscopic quantification or classification. Cross validation or independent validation should always be used to determine the model complexity. We recommend being highly conservative in choosing the number of LVs in the model to efficiently avoid overfitting the data.

\section{Non-negativity Least Squares (NNLS)}

Non-negativity least squares (NNLS) is an implementation of regression analysis with an imposed constraint to avoid negative abundances of biomolecules. Regression is typically performed against a library of Raman spectra measured from purified biomolecules (Haka et al., 2006). This allows one to estimate the concentration of the respective biomolecules. In general, care should be taken in regard to NNLS interpretations since these techniques require prior information of ECM components, which may not fully account for the molecular complexity present in the tissue.

\section{Spectral Unmixing}

Another type of computational analysis is the so called spectral unmixing techniques adapted from satellite imagery. These techniques seek to find the purest pixels in the hyperspectral Raman image using various implementations, including vertex component analysis (VCA) or N-finder (N-FINDR) (Hedegaard et al., 2016). Regression analysis is then applied to these pure spectra in order to extract the abundance of biomolecules in the tissue. Prior information in terms of reference spectra of pure chemicals is advantageous for correlation with the spectral signatures.

\section{Classification}

For classification purposes, a broad range of machine learning algorithms can be applied. For instance, for diagnostic tissue measurements, techniques such as LDA (Hutchings et al., 2010), partial least squares discriminant analysis (PLS-DA) (Bergholt et al., 2014), support vector machines (SVM) (Widjaja et al., 2008), and classification and regression trees (CART) (Teh et al., 2008) have been used. Many of these algorithms require determination of model complexity and should generally be assessed either through independent cross validation or a validation set.

All the above algorithms can be found in the implementations or inbuilt toolboxes in the Matlab, Python, and R scripting environments.

\section{RAMAN SPECTROSCOPY OF THE EXTRACELLULAR MATRIX}

\section{Cartilage Extracellular Matrix}

The organization of articular cartilage ECM is critical for its mechanical functionality. The cartilage ECM consists of collagen fibers interspersed with GAG macromolecules, which vary in concentration and alignment through the depth of the tissue, giving rise to its requisite mechanical and tribological behavior (Helen et al., 1970). During the onset of degenerative conditions, such as osteoarthritis (OA), the cartilage ECM is disrupted, contributing to the progressive degeneration of the tissue (Pearle et al., 2005). As such, OA research has long relied heavily on ECM characterization techniques. Interestingly, due to the strong Raman scattering properties of GAG and collagen, cartilage represents an ideal tissue for Raman spectroscopy applications. As outlined below, prior work on Raman-based cartilage ECM characterizations fall within the categories of (i) Raman microscopy imaging characterizations of the ECM distribution in tissue sections, and (ii) fiber-optic Raman assessments, aimed toward developing clinical intra-articular OA diagnostic platforms (Pavlou et al., 2018).

For work in Raman microscopy ECM characterizations, a recent study utilized NIR excitation to identify Raman bands that represented the presence of proteoglycans in both bone and cartilage tissues (Gamsjaeger et al., 2014). The analysis allowed for imaging of the ECM, yielding the distribution of GAG and type-II collagen in these tissues. Bonifacio et al. (2010) further used $632 \mathrm{~nm}$ laser excitation to generate images of the distribution of collagen and GAG across cartilage tissues using both PCA and PLS. In our own study of articular cartilage imaging, we performed confocal Raman microscopy using $532 \mathrm{~nm}$ laser excitation to obtain biomolecular and structural insights into the zonal complexity of articular cartilage. Using univariate and multivariate analysis (MCR), we extracted and quantified the collagen, GAG, and water signals of the ECM. Examples of univariate Raman images of bovine articular cartilage cross sections are shown in Figure 3. High resolution imaging showed that it is further possible to image the ECM distribution of the chondrocyte pericellular matrix. This study further demonstrated the advantage of using $532 \mathrm{~nm}$ laser excitation in a confocal setup to increase signal and reduce acquisition times, relative to NIR excitation.

Over the past decade, there has been growing interest in the potential applicability of fiber-optic Raman spectroscopy to offer novel advances in OA diagnostics. Given the limitations in current $\mathrm{OA}$ diagnostic technologies in terms of precision and instrumentation costs (Hayashi et al., 2016), the development of intra-articular fiber-optic Raman probes can potentially serve as a revolutionary OA diagnostic platform with particular utility in assessments of the critical early stages of the disease. Investigations into this avenue have focused predominantly on initial characterizations of degenerated cartilage on microscopybased systems, serving as a precursor to the development of more sophisticated fiber-optic platforms required for clinical diagnostics. In an early study, Lim et al. (2011) used a $785 \mathrm{~nm}$ Raman microscope to show that impact-injured porcine cartilage explants exhibit changes to the pyranose $1,126 \mathrm{~cm}^{-1}$ band intensity and the band area ratio of proline $\left(856 \mathrm{~cm}^{-1}\right)$ to hydroxyproline $\left(875 \mathrm{~cm}^{-1}\right)$, representing the GAG signal and collagen stability, respectively. Takahashi et al. (2014) performed Raman spectroscopic assessments with $647 \mathrm{~nm}$ excitation on human OA cartilage from knee arthroplasty procedures. Results demonstrated a host of peak intensity changes that varied with OA grade, notably an increase in the amide III band ratio of $1,241-1,269 \mathrm{~cm}^{-1}$ with OA. Kumar et al. also analyzed human OA cartilage using confocal Raman spectroscopy and 


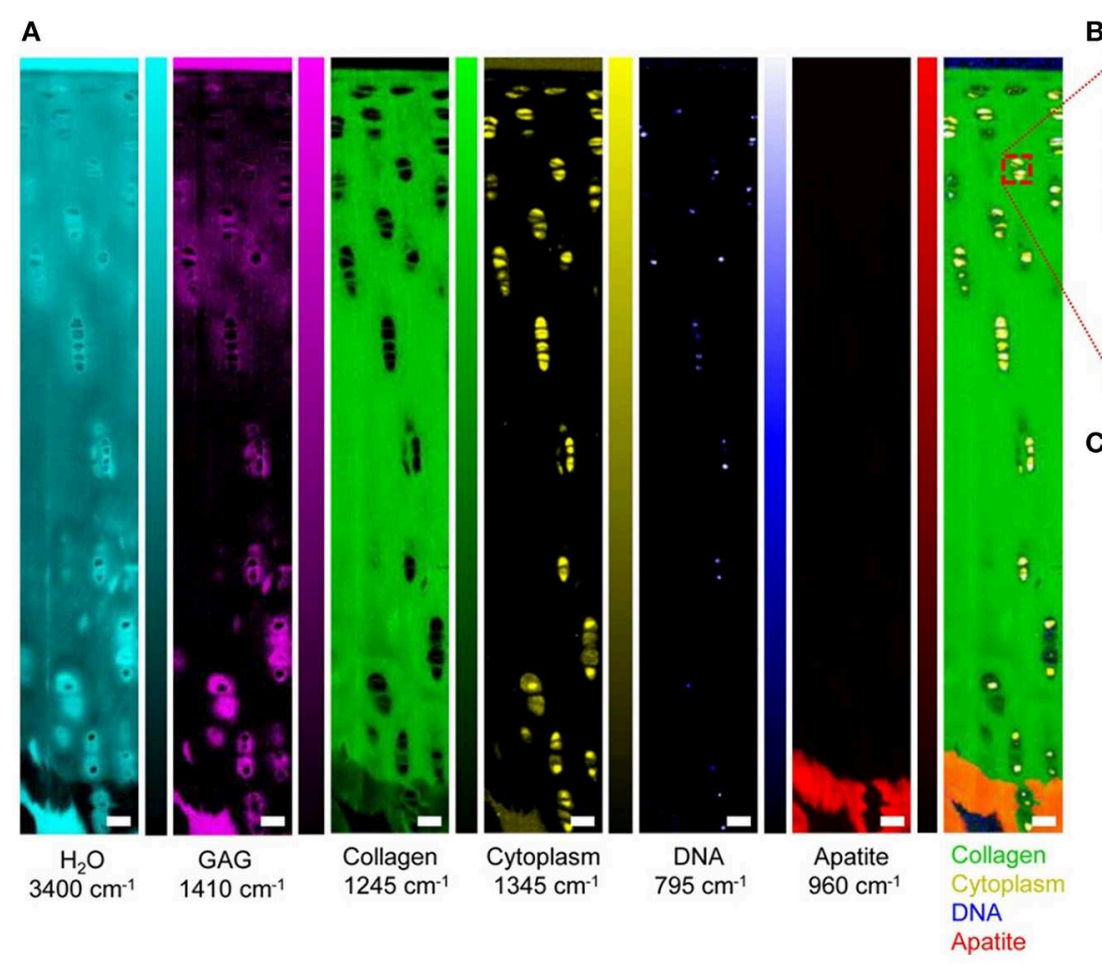

B

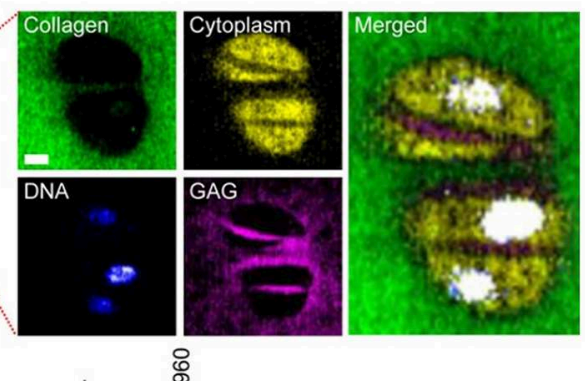

C

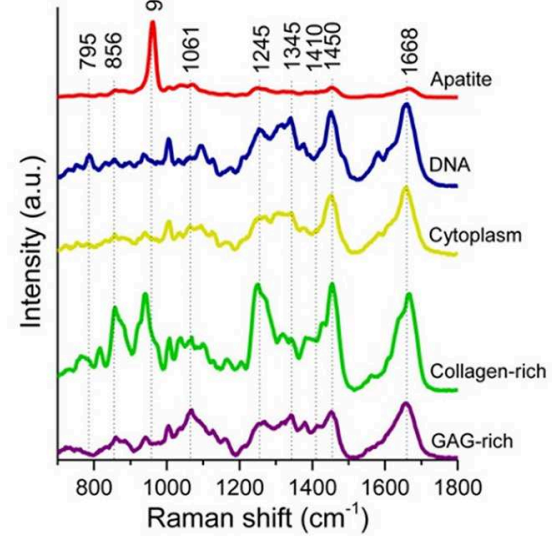

FIGURE 3 | [Reprinted with permission from Bergholt et al. (2016a)]. Raman spectroscopic imaging of articular cartilage. (A) Univariate Raman spectroscopy images of articular cartilage showing the band intensity associated with $\mathrm{H}_{2} \mathrm{O}\left(3,400 \mathrm{~cm}^{-1}\right), \mathrm{GAG}\left(1,410 \mathrm{~cm}^{-1}\right)$, collagen $\left(1,245 \mathrm{~cm}^{-1}\right)$, cytoplasm (1,345 cm $\left.{ }^{-1}\right)$, DNA (795 $\left.\mathrm{cm}^{-1}\right)$, apatite $\left(960 \mathrm{~cm}^{-1}\right)$, and the overlay. Scale bar: $50 \mu \mathrm{m}$. (B) High-resolution $(\sim 0.3 \mu \mathrm{m})$ Raman spectroscopy image of chondrocytes and pericellular matrix obtained by imaging the GAG $\left(1,410 \mathrm{~cm}^{-1}\right)$, cytoplasm $\left(1,345 \mathrm{~cm}^{-1}\right)$, and DNA $\left(795 \mathrm{~cm}^{-1}\right)$ against collagen $\left(1,245 \mathrm{~cm}{ }^{-1}\right)$. Scale bar: $3 \mu \mathrm{m}$. (C) Representative Raman spectra measured from articular cartilage with marked signatures for specific tissue components.

demonstrated sensitivity to different grades of the disease (Kumar et al., 2015). Tong et al. (2018) performed Raman spectral characterizations on porcine cartilage explants subjected to mechanical wear regimens, demonstrating a decrease in peak intensities corresponding to the protein backbone and amide bonds in collagen ( 817 and $1,670 \mathrm{~cm}^{-1}$ ). In an interesting novel analytical approach, Unal et al. (2019) recently demonstrated that analysis of high wavenumber Raman spectroscopy $(2,800$ to $3,800 \mathrm{~cm}^{-1}$ ) can be used to detect the water fractions in articular cartilage (bound versus unbound water) and the elevated tissue swelling associated with OA - results that were correlated with MRI measurements. In an example of the implementation of fiber-optic Raman spectroscopy, Esmonde-White et al. (2011) demonstrated that a fiber-optic probe can be used to assess cartilage thickness and mineralization degree in cadaver knee joint tissues. Further, de Souza et al. (2014) used a fiber-optic Raman spectroscopic probe to demonstrate increases in Raman ratios related to mineralization and tissue remodeling in an $\mathrm{OA}$ induced rat model. Overall, these investigations demonstrate that definitive spectral signature changes do occur in degenerating OA cartilage, strongly supporting the use of Raman spectral probes for molecular ECM analysis and degenerative diagnostics.

\section{Bone Extracellular Matrix}

Bone is a highly complex tissue comprised of two major structural ECM components: crystals of apatite and collagen fibrils arranged in a hierarchical structure. Raman spectroscopy has extensively been used to analyze how changes in bone composition and structure influence tissue-level bone mechanical properties (Draper et al., 2005; Mandair and Morris, 2015). Raman spectroscopy offers highly detailed information on both the bone mineral as well as the collagen matrix components (Raghavan et al., 2010). Due to the highly crystalline structure of bone, this tissue exhibits very strong Raman scattering. The highly intense phosphate mineral band near $\sim 960 \mathrm{~cm}^{-1}\left(\nu_{1} \mathrm{PO}_{4}^{3-}\right)$ is characteristic of carbonated apatites and is the most commonly used marker for bone tissue.

Raman techniques for bone assessment range from microscopy of bone tissue to fiber-optic and computed tomographic (CT) reconstruction approaches (Demers et al., 2012, 2015; Shu et al., 2018). A variety of applications have been reported, ranging from diagnostics of age-related bone loss (Donnelly et al., 2010), osteogenesis imperfecta (Imbert et al., 2014), osteoporosis, and the effect of therapeutic agents (Gamsjaeger et al., 2011, 2013; Olejnik et al., 2014). As an example of recent fiber-optic techniques, Shu et al. developed a spatial offset Raman spectroscopy (SORS) approach in order to non-invasively measure the bone signal in vivo from rodents (Shu et al., 2018). The SORS concept is based on diffuse light scattering and the principle that Raman scattered photons originating deep from the tissue can be detected as spatially offset from the excitation light while the shallow Raman photons 
can be detected closer to the excitation light (Matousek et al., 2005; Matousek and Stone, 2016). For instance in one SORS study on bone strength, Raman spectra were measured from the tibiae of mice aged 4-23 weeks old (Shu et al., 2018). PLS regression was then used to generate predictions of the areal bone mineral density ( $\mathrm{aBMD}$ ), volumetric bone mineralization density (vBMD), and maximum torque (MT) of each tibia as quantified by dual-energy X-ray absorptiometry, microCT imaging, and biomechanical tests, respectively. The authors found significant correlations between Raman spectral predictions and the reference values in all three categories, indicating that the fiber-optic Raman technique has great potential for diagnosing bone diseases, such as osteoporosis.

A range of novel fiber-optic Raman techniques based on diffuse Raman CT techniques has also been developed for transcutaneous imaging (Schulmerich et al., 2008a; Demers et al., 2012, 2015). These techniques have great potential for characterization of bone and tissue in vivo. For instance, Schulmerich et al. (2008b) applied a Raman CT to image canine bone tissue. Altogether, Raman spectroscopy represents a particularly powerful approach for bone ECM characterization due to the intense Raman signal originating from the ECM minerals and can therefore be applied across a broad range of applications.

\section{Cancer Extracellular Matrix}

Cancers are associated with abnormalities to the ECM. Destruction of the ECM barriers allow cancer cells to migrate from their in-situ location and metastasize systematically. Structural and biomolecular imaging of the ECM is, therefore, particularly important for shedding light on cancer diseases and developing novel therapeutics. Raman spectroscopy and imaging of cancers is an exciting and vibrant field; the examples are numerous and are continuously growing. A large number of studies have used Raman spectroscopy and imaging as an exploratory tool to characterize both the ECM and cells of cancer tissue across many organs, including the oral cavity (Barroso et al., 2016), skin (Kong et al., 2013), breast (Kong et al., 2014), prostate (Aubertin et al., 2018), lung (Huang et al., 2003b), esophagus (Kendall et al., 2003), gastric (Bergholt et al., 2014), and colon (Stone et al., 2004; Brauchle et al., 2018). Common across these studies is that cancer tissue shows a highly distinct spectral signature originating from the ECM and cells. As an example, Raman spectroscopy can be used to monitor how epithelial cells respond to signals from the ECM and, in particular, how cells lose their normal interactions with the ECM during cancer progression (Surmacki et al., 2013). For instance, Paidi used Raman imaging to evaluate pre-metastatic lungs and identified collagenous stroma and its cross-linkers, as well as proteoglycans (Paidi et al., 2017). They associated this with the metastatic potential of the primary tumor by recapitulating the compositional changes in the lungs. The correlated Raman results, histological assessment, and gene expression analysis suggested that remodeling of the extracellular matrix components may present promising markers for objective recognition of the pre-metastatic niche.

\section{Cardiovascular Extracellular Matrix}

In the cardiovascular system, the arterial wall is composed of a complex network of elastic and collagenous fibers. Imaging of the cardiovascular ECM is essential in order to understand pathogenesis of diseases, such as atherosclerosis. Recently, extensive work has been performed on Raman microscopy imaging of vascular tissues, including aorta and heart tissues (Manoharan et al., 1992). Using Raman and fluorescence spectral microscopy, one study examined the composition of ceroid in situ in aorta and coronary artery plaques (Haka et al., 2011). The combination of fluorescence and Raman spectroscopy allowed for identification of ceroid via its fluorescence signature and elucidation of its chemical composition using Raman spectroscopy. Reference chemicals, including collagen, used to extract quantitative information about the tissue. In another study, Votteler et al. (2012) employed NIR Raman spectroscopy on the ECM of heart valves to detect degrees of collagen degradation. In a Raman imaging study, You et al. (2017) used $785 \mathrm{~nm}$ confocal Raman microscopy imaging and VCA to elucidate the differences in medial aortic calcification and atherosclerosis. They identified the major ECM macromolecules of the aorta (i.e., elastin/collagen) and quantified the spatial distribution of minerals and lipids across the wall thickness (Figure 4). Further, this study found that whitlockite was highly associated with calcification of the aorta. This study highlighted that confocal Raman microscopy in combination with spectral unmixing is particularly advantageous for imaging the complex layers of vascular tissues.

\section{Extracellular Matrix Deposition in Tissue Engineering}

In recent years, Raman spectroscopy has served as a growing tool in the field of tissue engineering (TE) (Ember et al., 2017; Querido et al., 2017). TE represents an exciting multidisciplinary field that aims to generate live, functional replacement tissues (e.g., cardiac, bone, connective tissues) to treat a range of pathological disorders. A central challenge of TE is the generation of tissues that can recapitulate native ECM organizations in order to optimize functionality and performance upon native implantation (O'Connell et al., 2012). In this context, Raman spectroscopy can serve as an important quality assessment tool. Raman microscopy imaging can measure ECM distributions in engineered tissue sections in order to assess and guide the development of TE protocols. Further, fiber-optic Raman spectroscopic probes can serve as a clinical analytical tool, allowing for non-destructive, online monitoring of engineered tissue growth. Here, fiber-optic probes can be employed in vivo to monitor the development of engineered tissues after they have been implanted in the native environment. Further, probes can be employed in vitro to monitor the development of tissues during initial culture phases, allowing clinicians to select high-quality engineered tissue for implantation.

In a study measuring heterogeneities of ECM in engineered cartilage, we used $532 \mathrm{~nm}$ confocal Raman microscopy to image the distribution of newly deposited ECM in chondrocyte-seeded agarose-based cartilage tissue constructs (Figure 5). Here, we 


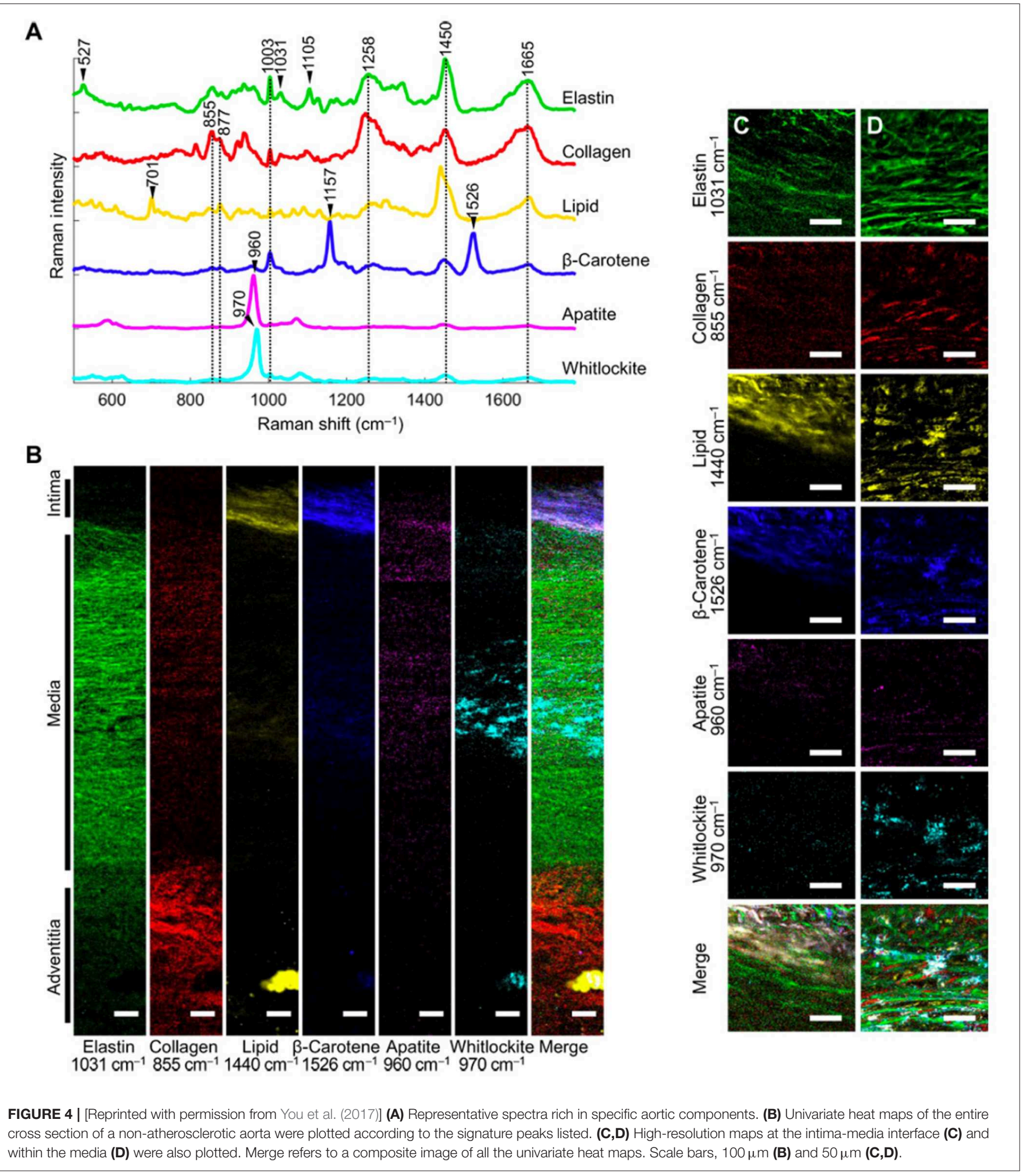

demonstrated that Raman imaging can achieve highly accurate, semi-quantitative measurements of the spatial distribution of GAG and collagen throughout engineered cartilage tissues, as evidenced by excellent agreement with profiles independently acquired via direct biochemical assaying of discrete spatial tissue sections. This validation serves as a foundational support for the exceptional quantitative nature of Raman image acquisitions. Further, in this study, Raman images were used to quantify the 

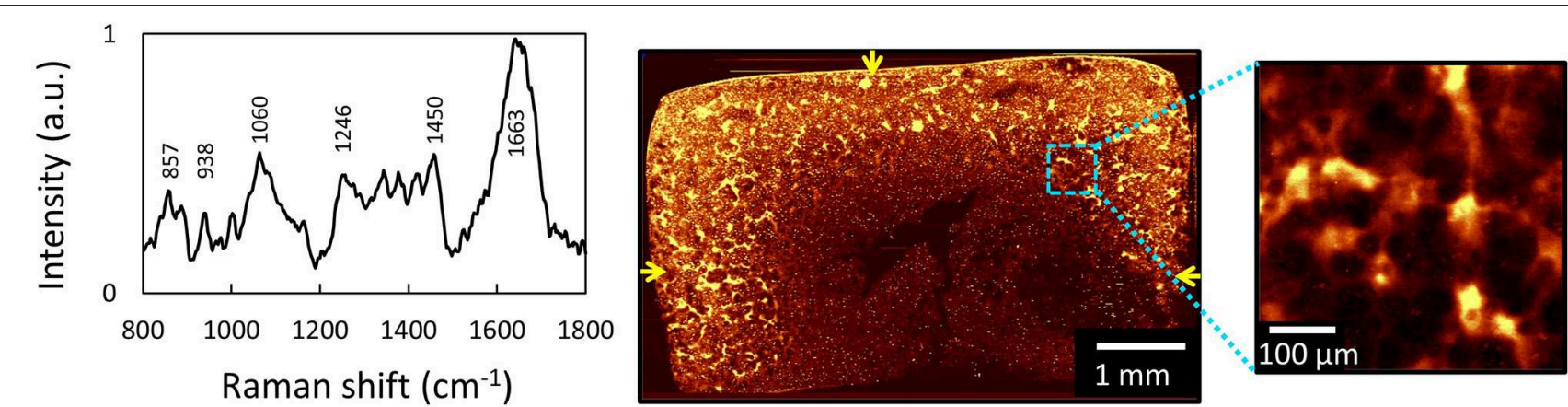

FIGURE 5 | [Reprinted and modified with permission from Albro et al. (2018)] (A) Raw spectra of engineered cartilage tissue from chondrocyte-seeded agarose hydrogel scaffolds. Raman spectroscopic image (univariate analysis) of ECM heterogeneities in 56-day cultured large engineered cartilage tissue construct (Ã $6 \times$ $3 \mathrm{~mm}$ ) for (B) the full cross-section (10 $\mu \mathrm{m}$ spatial resolution) and $\mathbf{( C )}$ a localized peripheral region (1 $\mu \mathrm{m}$ resolution). Arrows represent media-exposed surfaces.

microstructural heterogeneity of ECM in engineered cartilage, allowing for the generation of quantitative comparisons of the quality of local ECM distributions to that of native articular cartilage.

For investigations into Raman spectroscopy-based online monitoring of engineered tissue development, Kunstar et al. (2013) showed that ECM deposition in polymeric scaffolds could be assessed using Raman microscopy. The authors identified specific markers corresponding to collagen and sulfated GAGs. Collagen synthesis was found to differ between chondrocyteseeded scaffolds generated from isolated cells and cellular microaggregates. Normalized band-area ratios for collagen content of isolated cell-seeded samples gradually decreased during a 21-day culture period, whereas collagen content of the microaggregate-seeded samples increased significantly. Further, Kunstar et al. (2013) also implemented a fiber-optic Raman probe configuration to collect Raman spectra from multiple pores inside scaffolds. This highlights the exciting avenue of fiber-optic Raman spectral measurements for achieving highly valuable, non-destructive assessments of growing engineered tissues.

In a subsequent study, we demonstrated the ability of an NIR excitation fiber-optic Raman probe to perform online measurements of the deposition of collagen and GAG during the in vitro culture phase of engineered cartilage (Figure 6). Here, we measured diffuse NIR fiber-optic Raman spectra from living chondrocyte-seeded agarose tissue constructs in culture over a 56-day period. MCR modeling enabled quantitative biochemical analysis of the TE constructs. Raman spectroscopy showed an exceptional correlation with direct biochemical assays for measurements of collagen $\left(\mathrm{R}^{2}=0.84\right)$ and GAG $\left(\mathrm{R}^{2}=0.86\right)$. Further, Raman measured ECM contents exhibited a strong correlation with construct mechanical properties, suggesting that these non-destructive assessments can be clinically implemented to predict tissue functionality. Encouragingly, this study further confirmed that exposure of live tissue constructs to high intensity $785 \mathrm{~nm}$ excitation light induced no loss of cell viability or depression of ECM biosynthesis rates, serving as critical support for the applicability of NIR Raman spectroscopy in live-tissue quality control assessments.
In another recent study, Marzi et al. (2019) used NIR Raman spectroscopy to image human smooth muscle cells in cardiovascular tissue engineering. They monitored ECM remodeling in tissue-engineered rings comprised of human smooth muscle cells (SMC). Further, they measured the phenotypic switching in cultured SMCs and identified the impact of different culture conditions on ECM remodeling in the tissue-engineered ring constructs. Altogether, there is broad and growing utility of Raman spectroscopy in the field of TE.

\section{Collagen Structural Assessments}

A major untapped potential of Raman spectroscopy is the development of techniques to measure subtle changes to the molecular structure of ECM constituents (e.g., denaturing, crosslinking) that are not readily discernible with immunological techniques. Perhaps the collagen matrix is the most intriguing ECM component from this perspective due to its presence in a complex arrangement of varying subtypes, alignment, secondary structure, and cross-linking states (Shoulders and Raines, 2009). These arrangements give rise to unique ECM properties that strongly modulate tissue functionality. As collagen organizational remodeling is a hallmark feature of many pathologies, the ability to assess these organizational differences is critical for Raman microscopy and in vivo diagnostic applications. Raman spectral techniques for discerning collagen organization are of growing interest, particularly due to the lack of robust biochemical analytical techniques that directly measure these organizational arrangements. In TE applications, Raman imaging of collagen can be used to identify the collagen subtypes being deposited in the ECM and, as such, serve to assess engineered tissue quality. For example, in cartilage TE, one would be able to measure the ratio of type-II to type-I collagen as a means of assessing the formation of hyaline vs. fibrous cartilage.

The collagen protein itself has a unique Raman spectrum that can easily be discriminated from most other proteins (You et al., 2017; Martinez et al., 2019) due to its fibrillar nature, as well as the abundance of collagen-specific amino acids, such 


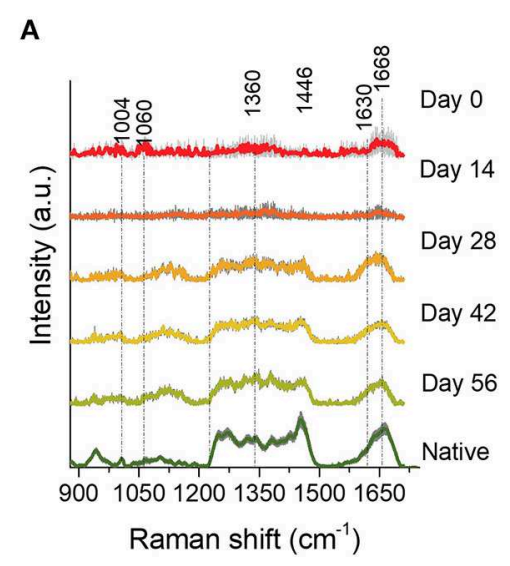

B

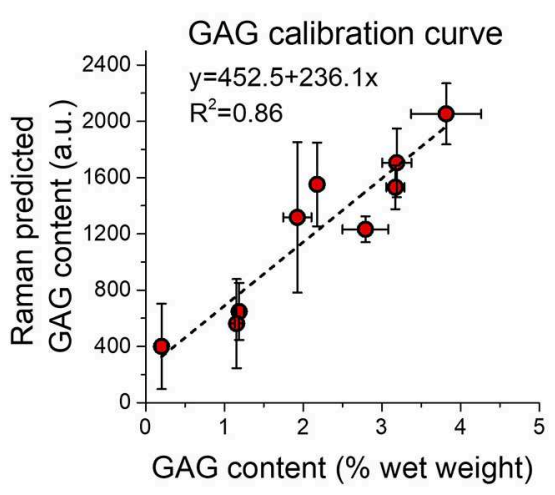

C

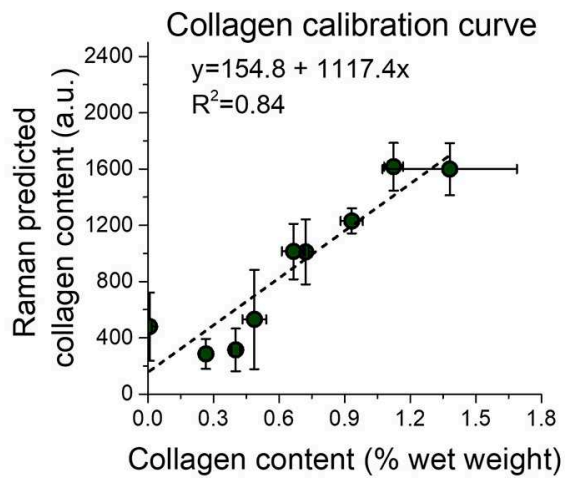

FIGURE 6 | Reprinted and modified with permission from Bergholt et al. (2017). Biochemically measured GAG and collagen contents of TE constructs after 0, 14, 28, 42, and 56 days of culture. (A) Mean Raman spectra \pm 1 standard deviation (SD) of native cartilage and TE constructs after varying culture durations. (B,C) Correlations between the Raman prediction (in arbitrary units) and biochemically measured GAG and collagen, respectively, with linear fits to the data. Raman spectroscopy showed an excellent correlation with biochemical assays for measurement of collagen $\left(R^{2}=0.84\right)$ and $G A G\left(R^{2}=0.86\right)$.

as hydroxyproline. However, discrimination between collagens of varying subtypes, secondary structure, cross-linking states, and alignment represents a considerable challenge in Raman assessments due to the subtle influence of these factors on the collagen spectral signature. Several studies have begun to explore this analytical capacity. For one, it has been shown that there are significant differences in the amide band between Type I and Type IV collagen (Nguyen et al., 2012). Dehring et al. (2006) demonstrated that changes in the alpha-helical stability of typeI collagen from aging can be assessed by the area ratio of the amide III bands in the $1,235 \mathrm{~cm}^{-1}$ position. Gamsjaeger et al. (2017) demonstrated that analysis of the Raman band 1,660 $\mathrm{cm}^{-1}$ wavenumbers can be used to assess pyridinoline crosslinks in animal tissue models. Fields et al. (2017) implemented a technique of high temperature Raman spectroscopy to assess the primary polypeptide structure of different collagen subtypes. Further, recent work has demonstrated that the utilization of Raman spectral measurements with polarized lasers can be used to measure the critical parameter of collagen alignment or anisotropy (Lim et al., 2011; Bergholt et al., 2016a). Overall, these recent characterizations have introduced the important potential for Raman spectroscopy to serve as a robust and comprehensive tool for providing collagen structural analysis in biological ECMs. However, the widespread adoption of Raman spectroscopy for collagen structural analysis will require expanded efforts to develop robust multivariate analytical models, and identify appropriate experimental model systems to validate the molecular specificity of Raman measurements.

\section{ADVANCED RAMAN METHODS}

\section{Non-linear Raman Imaging}

Several advanced Raman imaging approaches have emerged, such as coherent anti stokes Raman spectroscopy (CARS) and stimulated Raman scattering (SRS) microscopy (Camp et al.,
2014; Schie et al., 2015; Lu et al., 2016). These techniques are able to speed up image acquisition times relative to conventional Raman spectroscopy, but require the implementation of complex instrumentation, including multiphoton lasers. Examples of application to ECM imaging are numerous. For instance, Mansfield et al. (2013) applied stimulated Raman scattering (SRS) in order to image the interface of cartilage and bone with high molecular contrast. SRS represents an advanced Raman technique that offers high speed imaging and reveals high quality spectroscopic images of the peri- and extracellular matrix. Since Raman is highly sensitive to lipids, the SRS approach revealed highly localized lipids in the pericellular matrix of chondrocytes. Recent exciting implementations of CARS and SRS enable the full range of Raman spectra to be measured, opening the opportunities for rapid hyperspectral Raman imaging (Parekh et al., 2010). Very recently, more exotic nonlinear Raman techniques have emerged aiming toward label-free super-resolution imaging (Silva et al., 2016; Gong et al., 2019).

\section{Correlative Raman Spectroscopy and Imaging}

To complement and potentially advance Raman spectroscopic imaging beyond conventional analysis, correlative imaging is continually advancing. Raman imaging has been combined with a plurality of other techniques, such as fluorescence (Bennet et al., 2014), atomic force microscopy (Brauchle et al., 2018), nanoindentation (Bergholt et al., 2016a), mass spectrometry (Bergholt et al., 2018), optic coherence tomography (OCT) (Patil et al., 2011), and FT-IR imaging (Lasch and Noda, 2017). Raman spectroscopy has also been correlated with mechanical measurement techniques (Unal and Akkus, 2015). Among the advanced techniques, a vibrant and promising field is the implementation of heterospectral imaging approaches, which directly interrelate the hyperspectral data of several modalities at the pixel level for a more comprehensive biomolecular analysis (Lasch and Noda, 2017, 2019; Bergholt et al., 2018; 
Ryabchykov et al., 2018). For instance, Lasch and Noda (2017) and Lasch and Noda (2019) introduced multimodal FT-IR, Raman, and MALDI-TOF imaging for tissue characterization. An important part of correlative imaging is the lateral resolution matching between diffraction limited Raman imaging and the correlative modality. This resolution matching can efficiently be achieved through binning or interpolation (Lasch and Noda, 2017; Bergholt et al., 2018).

\section{Fiber-Optic Raman Spectroscopy}

The capability of Raman spectroscopy to offer label-free tissue characterizations has fuelled the field of fiber-optic Raman spectroscopy for clinical cancer detection and diagnostics (Shim et al., 2000; Utzinger and Richards-Kortum, 2003; Bergholt et al., 2014; Baker et al., 2018; McGregor et al., 2018). There have been extensive efforts in the development of miniaturized fiber-optic probes compatible with medical endoscopes in order to measure the biomolecular composition of tissues in vivo (Shim et al., 2000; Komachi et al., 2006). Since the clinical regulation pathway for label-free technologies is short relative to studies using exogenous contrast agents, there have been several large-scale human trials for in vivo tissue characterization and diagnostics. Key biomedical applications range from the in vivo diagnosis of diseases (Chau et al., 2008; Kendall et al., 2009; Pence et al., 2017) to the monitoring of the growth of engineered tissues (Bergholt et al., 2017). These techniques, in general, probe both the cells and ECM, and offer a compound Raman spectrum that carries information about the overall tissue composition. As an example of targeted in vivo analysis, the Mahadevan group recently performed an in vivo study of impaired cervical remodeling in a mouse model of delayed parturition (O'Brien et al., 2017). Deletion of cyclooxygenase-1 (Cox-1) results in delayed parturition in mice. They used Raman spectroscopy to detect spectral changes related to extracellular matrix proteins, lipids, and nucleic acids over pregnancy. They concluded that in vivo Raman spectroscopy non-invasively detected abnormal remodeling in the Cox-1 null mouse, and clearly demonstrated that the cervix plays a key role in their delayed parturition.

\section{Prospective and Conclusion}

Histological imaging has long served as the gold standard for visualizing specific molecular ECM constituents. Raman spectroscopy represents a label-free tool that offers an additional level of molecular information, with applicability for both microscopy-based characterizations and minimally invasive diagnostics of the ECM structure and composition.

\section{REFERENCES}

Albro, M. B., Bergholt, M. S., St-Pierre, J. P., Vinals Guitart, A., Zlotnick, H. M., Evita, E. G., et al. (2018). Raman spectroscopic imaging for quantification of depth-dependent and local heterogeneities in native and engineered cartilage. Regener. Med. 3:3. doi: 10.1038/s41536-018-0042-7

Aubertin, K., Desroches, J., Jermyn, M., Trinh, V. Q., Saad, F., Trudel, D., et al. (2018). Combining high wavenumber and fingerprint Raman spectroscopy for
There are still many challenges in the widespread adoption of Raman spectroscopy imaging in biomedicine, predominantly due to the technical difficulties in data interpretation and analysis. Mounting efforts must, therefore, be placed into streamlining Raman analysis through the development of protocols for correlative Raman spectroscopy and imaging. Further, more standardized preprocessing, data analysis techniques, and calibration transfer are needed in order to improve the reproducibility of results across laboratories. Furthermore, for Raman spectroscopic imaging to gain wider acceptance in biomedicine, Raman imaging of tissues should be correlated with appropriate reference methods, such as immunofluorescence or direct biochemical assay measurements. Future developments could include correlation of hyperspectral Raman images of the ECM with sophisticated molecular-based characterization approaches, such as proteomics.

In terms of analysis, besides conventional multivariate algorithms, we anticipate that Raman spectroscopy will begin to rely heavily on deep learning approaches that still remain in their early stages for Raman spectral analysis. Since deep learning relies on neural networks, the large datasets that Raman imaging provides could serve as an excellent data source. The use of deep learning can potentially be used for regression and classification, but also for Raman image resolution enhancement and correlative imaging.

Overall, Raman spectroscopy represents a powerful tool that provides a wealth of quantitative information about the molecular structure of a tissue's ECM. The potential application avenues for Raman spectroscopy in biomedicine are vast. In many respects, the field is still in its infancy and will continue to grow with the expansion of equipment technologies and analytical algorithms. We anticipate that in the near future, Raman spectroscopy can develop into one of the premiere technologies for tissue diagnostics and analytics.

\section{AUTHOR CONTRIBUTIONS}

All authors listed have made a substantial, direct and intellectual contribution to the work, and approved it for publication.

\section{FUNDING}

This work has received funding from the European Research Council (ERC) under the European Union's Horizon 2020 research and innovation programme (grant agreement No. 802778). This grant has allocated funding for open access publication.

the detection of prostate cancer during radical prostatectomy. Biomed. Opt. Express. 9, 4294-4305. doi: 10.1364/BOE.9.004294

Baker, M. J., Byrne, H. J., Chalmers, J., Gardner, P., Goodacre, R., Henderson, A., et al. (2018). Clinical applications of infrared and Raman spectroscopy: state of play and future challenges. Analyst 143, 1735-1757. doi: 10.1039/C7AN $01871 \mathrm{~A}$

Barroso, E. M., Smits, R. W., van Lanschot, C. G., Caspers, P. J., Ten Hove, I., Mast, H., et al. (2016). Water concentration analysis by Raman spectroscopy to 
determine the location of the tumor border in oral cancer surgery. Cancer Res. 76, 5945-5953. doi: 10.1158/0008-5472.CAN-16-1227

Bennet, M., Akiva, A., Faivre, D., Malkinson, G., Yaniv, K., Abdelilah-Seyfried, S., et al. (2014). Simultaneous Raman microspectroscopy and fluorescence imaging of bone mineralization in living zebrafish larvae. Biophys. J. 106, L17-L19. doi: 10.1016/j.bpj.2014.01.002

Bergholt, M. S., Albro, M. B., and Stevens, M. M. (2017). Online quantitative monitoring of live cell engineered cartilage growth using diffuse fiber-optic Raman spectroscopy. Biomaterials 140, 128-137. doi: 10.1016/j.biomaterials.2017.06.015

Bergholt, M. S., Lin, K., Wang, J., Zheng, W., Xu, H., Huang, Q., et al. (2016b). Simultaneous fingerprint and high-wavenumber fiber-optic Raman spectroscopy enhances real-time in vivo diagnosis of adenomatous polyps during colonoscopy. J. Biophotonics 9, 333-342. doi: 10.1002/jbio.201400141

Bergholt, M. S., Serio, A., McKenzie, J. S., Boyd, A., Soares, R. F., Tillner, J., et al. (2018). Correlated heterospectral lipidomics for biomolecular profiling of remyelination in multiple sclerosis. ACS Central Sci. 4, 39-51. doi: 10.1021/acscentsci.7b00367

Bergholt, M. S., St-Pierre, J. P., Offeddu, G. S., Parmar, P. A., Albro, M. B., Puetzer, J. L., et al. (2016a). Raman spectroscopy reveals new insights into the zonal organization of native and tissue-engineered articular cartilage. ACS Central Sci. 2, 885-895. doi: 10.1021/acscentsci.6b00222

Bergholt, M. S., Zheng, W., Ho, K. Y., Teh, M., Yeoh, K. G., Yan So, J. B., et al. (2014). Fiberoptic confocal raman spectroscopy for real-time in vivo diagnosis of dysplasia in Barrett's esophagus. Gastroenterology 146, 27-32. doi: 10.1053/j.gastro.2013.11.002

Bonifacio, A., Beleites, C., Vittur, F., Marsich, E., Semeraro, S., Paoletti, S., et al. (2010). Chemical imaging of articular cartilage sections with Raman mapping, employing uni- and multi-variate methods for data analysis. Analyst 135, 3193-3204. doi: 10.1039/c0an00459f

Bonnans, C., Chou, J., and Werb, Z. (2014). Remodelling the extracellular matrix in development and disease. Nat. Rev. Mol. Cell Biol. 15:786. doi: $10.1038 / \mathrm{nrm} 3904$

Brauchle, E., Kasper, J., Daum, R., Schierbaum, N., Falch, C., Kirschniak, A., et al. (2018). Biomechanical and biomolecular characterization of extracellular matrix structures in human colon carcinomas. Matrix Biol. 68-69, 180-93. doi: 10.1016/j.matbio.2018.03.016

Brightman, A. O., Rajwa, B. P., Sturgis, J. E., McCallister, M. E., Robinson, J. P., and Voytik-Harbin, S. L. (2000). Time-lapse confocal reflection microscopy of collagen fibrillogenesis and extracellular matrix assembly in vitro. Biopolymers 54, 222-234. doi: 10.1002/1097-0282(200009)54:3<222::AID-BIP80>3.0.CO2-K;

Burke, K., Tang, P., and Brown, E. (2013). Second harmonic generation reveals matrix alterations during breast tumor progression. J. Biomed. Opt. 18:31106. doi: 10.1117/1.JBO.18.3.031106

Camp, C. H. Jr., Lee, Y. J., Heddleston, J. M., Hartshorn, C. M., Hight Walker, A. R., Rich, J. N., et al. (2014). High-speed coherent Raman fingerprint imaging of biological tissues. Nat. Photonics 8, 627-634. doi: 10.1038/nphoton.2014.145

Chahine, N. O., Wang, C. C. B., Hung, C. T., and Ateshian, G. A. (2004). Anisotropic strain-dependent material properties of bovine articular cartilage in the transitional range from tension to compression. J. Biomech. 37, 1251-1261. doi: 10.1016/j.jbiomech.2003.12.008

Chau, A. H., Motz, J. T., Gardecki, J. A., Waxman, S., Bouma, B. E., and Tearney, G. J. (2008). Fingerprint and high-wavenumber Raman spectroscopy in a human-swine coronary xenograft in vivo. J. Biomed. Opt. 13:040501. doi: $10.1117 / 1.2960015$

Cheheltani, R., McGoverin, C. M., Rao, J., Vorp, D. A., Kiani, M. F., and Pleshko, N. (2014). Fourier transform infrared spectroscopy to quantify collagen and elastin in an in vitro model of extracellular matrix degradation in aorta. Analyst 139, 3039-3047. doi: 10.1039/C3AN02371K

Cocciolone, A. J., Hawes, J. Z., Staiculescu, M. C., Johnson, E. O., Murshed, M., and Wagenseil, J. E. (2018). Elastin, arterial mechanics, and cardiovascular disease. Am. J. Physiol. Heart Circ. Physiol. 315, H189-H205. doi: 10.1152/ajpheart.00087.2018

Czamara, K., Majzner, K., Pacia, M. Z., Kochan, K., Kaczor, A., and Baranska, M. (2015). Raman spectroscopy of lipids: a review. J. Raman Spectrosc. 46, 4-20. doi: $10.1002 /$ jrs. 4607 de Souza, R. A., Xavier, M., Mangueira, N. M., Santos, A. P., Pinheiro, A. L., Villaverde, A. B., et al. (2014). Raman spectroscopy detection of molecular changes associated with two experimental models of osteoarthritis in rats. Lasers Med. Sci. 29, 797-804. doi: 10.1007/s10103-013-1423-1

Dehring, K. A., Smukler, A. R., Roessler, B. J., and Morris, M. D. (2006). Correlating changes in collagen secondary structure with aging and defective type II collagen by Raman spectroscopy. Appl. Spectrosc. 60, 366-372. doi: 10.1366/000370206776593582

Demers, J.-L. H., Esmonde-White, F. W. L., Esmonde-White, K. A., Morris, M. D., and Pogue, B. W. (2015). Next-generation Raman tomography instrument for non-invasive in vivo bone imaging. Biomed. Opt. Express. 6, 793-806. doi: 10.1364/BOE.6.000793

Demers, J. L., Davis, S. C., Pogue, B. W., and Morris, M. D. (2012). Multichannel diffuse optical Raman tomography for bone characterization in vivo: a phantom study. Biomed. Opt. Express. 3, 2299-2305. doi: 10.1364/BOE.3.002299

Donnelly, E., Boskey, A. L., Baker, S. P., and van der Meulen, M. C. (2010). Effects of tissue age on bone tissue material composition and nanomechanical properties in the rat cortex. J. Biomed. Mater. Res. Part A 92, 1048-1056. doi: 10.1002/jbm.a.32442

Draper, E. R., Morris, M. D., Camacho, N. P., Matousek, P., Towrie, M., Parker, A. W., et al. (2005). Novel assessment of bone using time-resolved transcutaneous Raman spectroscopy. J. Bone Mineral Res. 20, 1968-1972. doi: 10.1359/JBMR.050710

Duffield, J. S. (2014). Cellular and molecular mechanisms in kidney fibrosis. J. Clin. Investig. 124, 2299-2306. doi: 10.1172/JCI72267

Ember, K. J. I., Hoeve, M. A., McAughtrie, S. L., Bergholt, M. S., Dwyer, B. J., Stevens, M. M., et al. (2017). Raman spectroscopy and regenerative medicine: a review. npj Regen. Med. 2:12. doi: 10.1038/s41536-017-0014-3

Esmonde-White, K. (2014). Raman spectroscopy of soft musculoskeletal tissues. Appl. Spectrosc. 68, 1203-1218. doi: 10.1366/14-07592

Esmonde-White, K. A., Esmonde-White, F. W., Morris, M. D., and Roessler, B. J. (2011). Fiber-optic Raman spectroscopy of joint tissues. Analyst 136, 1675-1685. doi: 10.1039/c0an00824a

Fields, M., Spencer, N., Dudhia, J., and McMillan, P. F. (2017). Structural changes in cartilage and collagen studied by high temperature Raman spectroscopy. Biopolymers 107:e23017. doi: 10.1002/bip.23017

Gaifulina, R., Maher, A. T., Kendall, C., Nelson, J., Rodriguez-Justo, M., Lau, K., et al. (2016). Label-free Raman spectroscopic imaging to extract morphological and chemical information from a formalin-fixed, paraffin-embedded rat colon tissue section. Int. J. Exp. Pathol. 97, 337-350. doi: 10.1111/iep. 12194

Gamsjaeger, S., Buchinger, B., Zoehrer, R., Phipps, R., Klaushofer, K., and Paschalis, E. P. (2011). Effects of one year daily teriparatide treatment on trabecular bone material properties in postmenopausal osteoporotic women previously treated with alendronate or risedronate. Bone 49, 1160-1165. doi: 10.1016/j.bone.2011.08.015

Gamsjaeger, S., Hofstetter, B., Zwettler, E., Recker, R., Gasser, J. A., Eriksen, E. F., et al. (2013). Effects of 3 years treatment with once-yearly zoledronic acid on the kinetics of bone matrix maturation in osteoporotic patients. Osteoporosis Int. 24, 339-347. doi: 10.1007/s00198-012-2202-8

Gamsjaeger, S., Klaushofer, K., and Paschalis, E. P. (2014). Raman analysis of proteoglycans simultaneously in bone and cartilage. J. Raman Spectrosc. 45, 794-800. doi: 10.1002/jrs.4552

Gamsjaeger, S., Robins, S. P., Tatakis, D. N., Klaushofer, K., and Paschalis, E. P. (2017). Identification of pyridinoline trivalent collagen crosslinks by Raman microspectroscopy. Calcified Tissue Int. 100, 565-574. doi: 10.1007/s00223-016-0232-5

Gessel, M., Spraggins, J. M., Voziyan, P., Hudson, B. G., and Caprioli, R. M. (2015). Decellularization of intact tissue enables MALDI imaging mass spectrometry analysis of the extracellular matrix. J. Mass Spectrometry. 50, 1288-1293. doi: $10.1002 /$ jms.3696

Gilkes, D. M., Semenza, G. L., and Wirtz, D. (2014). Hypoxia and the extracellular matrix: drivers of tumour metastasis. Nat. Rev. Cancer 14, 430-439. doi: 10.1038/nrc3726

Gong, L., Zheng, W., Ma, Y., and Huang, Z. (2019). Saturated stimulated-Ramanscattering microscopy for far-field superresolution vibrational imaging. Phys. Rev. Appl. 11:034041. doi: 10.1103/PhysRevApplied.11.034041 
Grauw, C. J. D., Otto, C., and Greve, J. (1997). Line-scan Raman microspectrometry for biological applications. Appl. Spectrosc. 51, 1607-1612. doi: 10.1366/0003702971939587

Haka, A. S., Kramer, J. R., Dasari, R. R., and Fitzmaurice, M. (2011). Mechanism of ceroid formation in atherosclerotic plaque: in situ studies using a combination of Raman and fluorescence spectroscopy. J. Biomed. Opt. 16:011011. doi: 10.1117/1.3524304

Haka, A. S., Volynskaya, Z., Gardecki, J. A., Nazemi, J., Lyons, J., Hicks, D., et al. (2006). In vivo margin assessment during partial mastectomy breast surgery using Raman spectroscopy. Cancer Res. 66, 3317-3322. doi: 10.1158/0008-5472.CAN-05-2815

Hayashi, D., Roemer, F. W., and Guermazi, A. (2016). Imaging for osteoarthritis. Ann. Phys. Rehabil. Med. 59, 161-169. doi: 10.1016/j.rehab.2015.12.003

Hedegaard, M. A., Bergholt, M. S., and Stevens, M. M. (2016). Quantitative multiimage analysis for biomedical Raman spectroscopic imaging. J. Biophotonics 9, 542-550. doi: 10.1002/jbio.201500238

Helen, M., Peter, B., and Alice, M. (1970). The distribution of collagen in human articular cartilage with some of its physiological implications. J. Bone Joint Surg. Br. 52B, 554-563. doi: 10.1302/0301-620X.52B3.554

Huang, N., Short, M., Zhao, J., Wang, H., Lui, H., Korbelik, M., et al. (2011). Full range characterization of the Raman spectra of organs in a murine model. Opt Express. 19, 22892-22909. doi: 10.1364/OE.19.022892

Huang, Z., McWilliams, A., Lam, S., English, J., McLean, D. I., Lui, H., et al. (2003a). Effect of formalin fixation on the near-infrared Raman spectroscopy of normal and cancerous human bronchial tissues. Int. J. Oncol. 23, 649-655. doi: 10.3892/ijo.23.3.649

Huang, Z., McWilliams, A., Lui, H., McLean, D. I., Lam, S., and Zeng, H. (2003b). Near-infrared Raman spectroscopy for optical diagnosis of lung cancer. Int. J. Cancer 107, 1047-1052. doi: 10.1002/ijc.11500

Hutchings, J., Kendall, C., Shepherd, N., Barr, H., and Stone, N. (2010). Evaluation of linear discriminant analysis for automated Raman histological mapping of esophageal high-grade dysplasia. J. Biomed. Opt. 15:066015. doi: $10.1117 / 1.3512244$

Hyllested, J. L., Veje, K., and Ostergaard, K. (2002). Histochemical studies of the extracellular matrix of human articular cartilage-a review. Osteoarthritis Cartilage 10, 333-343. doi: 10.1053/joca.2002.0519

Imbert, L., Auregan, J. C., Pernelle, K., and Hoc, T. (2014). Mechanical and mineral properties of osteogenesis imperfecta human bones at the tissue level. Bone 65, 18-24. doi: 10.1016/j.bone.2014.04.030

Janko, M., Davydovskaya, P., Bauer, M., Zink, A., and Stark, R. W. (2010). Anisotropic Raman scattering in collagen bundles. Opt Lett. 35, 2765-2767. doi: 10.1364/OL.35.002765

Jorba, I., Uriarte, J. J., Campillo, N., Farre, R., and Navajas, D. (2017). Probing micromechanical properties of the extracellular matrix of soft tissues by atomic force microscopy. J. Cell. Physiol. 232, 19-26. doi: 10.1002/jcp.25420

Jubb, R. W., and Eggert, F. M. (1981). Staining of demineralized cartilage. Histochemistry 73, 391-396. doi: 10.1007/BF00495654

Jung, E., Choi, H. J., Lim, M., Kang, H., Park, H., Han, H., et al. (2012a). Quantitative analysis of water distribution in human articular cartilage using terahertz time-domain spectroscopy. Biomed. Opt. Express. 3, 1110-1115. doi: 10.1364/BOE.3.001110

Jung, E., Park, H., Moon, K., Lim, M., Do, Y., Han, H., et al. (2012b). $\mathrm{THz}$ time-domain spectroscopic imaging of human articular cartilage. J. Infrared Millimeter Terahertz Waves 33, 593-598. doi: 10.1007/s10762-0129903-0

Kallepitis, C., Bergholt, M. S., Mazo, M. M., Leonardo, V., Skaalure, S. C., Maynard, S. A., et al. (2017). Quantitative volumetric Raman imaging of three dimensional cell cultures. Nat. Commun. 8:14843. doi: 10.1038/ncomms14843

Kendall, C., Isabelle, M., Bazant-Hegemark, F., Hutchings, J., Orr, L., Babrah, J., et al. (2009). Vibrational spectroscopy: a clinical tool for cancer diagnostics. Analyst 134, 1029-1045. doi: 10.1039/b822130h

Kendall, C., Stone, N., Shepherd, N., Geboes, K., Warren, B., Bennett, R., et al. (2003). Raman spectroscopy, a potential tool for the objective identification and classification of neoplasia in Barrett's oesophagus. J. Pathol. 200, 602-609. doi: $10.1002 /$ path.1376

Kisseleva, T. (2017). The origin of fibrogenic myofibroblasts in fibrotic liver. Hepatology 65, 1039-1043. doi: 10.1002/hep.28948
Komachi, Y., Sato, H., and Tashiro, H. (2006). Intravascular Raman spectroscopic catheter for molecular diagnosis of atherosclerotic coronary disease. Appl. Opt. 45, 7938-7943. doi: 10.1364/AO.45.007938

Kong, K., Rowlands, C. J., Varma, S., Perkins, W., Leach, I. H., Koloydenko, A. A., et al. (2013). Diagnosis of tumors during tissue-conserving surgery with integrated autofluorescence and Raman scattering microscopy. Proc. Natl. Acad. Sci. U.S.A. 110, 15189-15194. doi: 10.1073/pnas.1311289110

Kong, K., Zaabar, F., Rakha, E., Ellis, I., Koloydenko, A., and Notingher, I. (2014). Towards intra-operative diagnosis of tumours during breast conserving surgery by selective-sampling Raman micro-spectroscopy. Phys. Med. Biol. 59, 6141-6152. doi: 10.1088/0031-9155/59/20/6141

Krishnan, R., Park, S., Eckstein, F., and Ateshian, G. A. (2003). Inhomogeneous cartilage properties enhance superficial interstitial fluid support and frictional properties, but do not provide a homogeneous state of stress. J. Biomech. Eng. 125, 569-577. doi: 10.1115/1.1610018

Krishnan, Y., and Grodzinsky, A. J. (2018). Cartilage diseases. Matrix Biol. 71-72, 51-69. doi: 10.1016/j.matbio.2018.05.005

Kuhar, N., Sil, S., Verma, T., and Umapathy, S. (2018). Challenges in application of Raman spectroscopy to biology and materials. RSC Adv. 8, 25888-25908. doi: 10.1039/C8RA04491K

Kumar, R., Grønhaug, K. M., Afseth, N. K., Isaksen, V., de Lange Davies, C., Drogset, J. O., et al. (2015). Optical investigation of osteoarthritic human cartilage (ICRS grade) by confocal Raman spectroscopy: a pilot study. Anal. Bioanal. Chem. 407, 8067-8077. doi: 10.1007/s00216-0158979-5

Kunstar, A., Leferink, A. M., Okagbare, P. I., Morris, M. D., Roessler, B. J., Otto, C., et al. (2013). Label-free Raman monitoring of extracellular matrix formation in three-dimensional polymeric scaffolds. J. R. Soc. Interface 10:20130464. doi: 10.1098/rsif.2013.0464

Lasch, P., and Noda, I. (2017). Two-Dimensional correlation spectroscopy for multimodal analysis of FT-IR, Raman, and MALDI-TOF MS hyperspectral images with hamster brain tissue. Anal. Chem. 89, 5008-5016. doi: 10.1021/acs.analchem.7b00332

Lasch, P., and Noda, I. (2019). Two-dimensional correlation spectroscopy (2DCOS) for analysis of spatially resolved vibrational spectra. Appl. Spectrosc. 73, 359-379. doi: 10.1177/0003702818819880

Leikin, S., Parsegian, V. A., Yang, W.-H., and Walrafen, G. E. (1997). Raman spectral evidence for hydration forces between collagen triple helices. Proc. Natl. Acad. Sci. U.S.A. 94, 11312-11317. doi: 10.1073/pnas.94.21.11312

Lieber, C. A., and Mahadevan-Jansen, A. (2003). Automated method for subtraction of fluorescence from biological Raman spectra. Appl. Spectrosc. 57, 1363-1367. doi: 10.1366/000370203322554518

Lim, N. S., Hamed, Z., Yeow, C. H., Chan, C., and Huang, Z. (2011). Early detection of biomolecular changes in disrupted porcine cartilage using polarized Raman spectroscopy. J. Biomed. Opt. 16:017003. doi: 10.1117/1.3528006

Lu, F.-K., Basu, S., Igras, V., Hoang, M. P., Ji, M., Fu, D., et al. (2015). Label-free DNA imaging in vivo with stimulated Raman scattering microscopy. Proc. Natl. Acad. Sci. U.S.A. 112, 11624-11629. doi: 10.1073/pnas.1515121112

Lu, F. K., Calligaris, D., Olubiyi, O. I., Norton, I., Yang, W., Santagata, S., et al. (2016). Label-free neurosurgical pathology with stimulated Raman imaging. Cancer Res. 76, 3451-3462. doi: 10.1158/0008-5472.CAN-16-0270

Mandair, G. S., and Morris, M. D. (2015). Contributions of Raman spectroscopy to the understanding of bone strength. Bonekey Rep. 4:620. doi: 10.1038/bonekey.2014.115

Manoharan, R., Baraga, J. J., Feld, M. S., and Rava, R. P. (1992). Quantitative histochemical analysis of human artery using Raman spectroscopy. J. Photochem. Photobiol. B Biol. 16, 211-233. doi: 10.1016/1011-1344(92)80009-k

Mansfield, J., Moger, J., Green, E., Moger, C., and Winlove, C. P. (2013). Chemically specific imaging and in-situ chemical analysis of articular cartilage with stimulated Raman scattering. J. Biophotonics 6, 803-814. doi: 10.1002/jbio.201200213

Martinez, M. G., Bullock, A. J., MacNeil, S., and Rehman, I. U. (2019). Characterisation of structural changes in collagen with Raman spectroscopy. Appl. Spectrosc. Rev. 54, 509-542. doi: 10.1080/05704928.2018. H1506799

Marzi, J., Brauchle, E. M., Schenke-Layland, K., and Rolle, M. W. (2019). Noninvasive functional molecular phenotyping of human smooth muscle cells 
utilized in cardiovascular tissue engineering. Acta Biomater. 89, 193-205. doi: 10.1016/j.actbio.2019.03.026

Matousek, P., Clark, I. P., Draper, E. R., Morris, M. D., Goodship, A. E., Everall, N., et al. (2005). Subsurface probing in diffusely scattering media using spatially offset Raman spectroscopy. Appl. Spectrosc. 59, 393-400. doi: 10.1366/0003702053641450

Matousek, P., and Stone, N. (2016). Development of deep subsurface Raman spectroscopy for medical diagnosis and disease monitoring. Chem. Soc. Rev. 45, 1794-1802. doi: 10.1039/C5CS00466G

McGregor, H. C., Short, M. A., Lam, S., Shaipanich, T., Beaudoin, E. L., and Zeng, H. (2018). Development and in vivo test of a miniature Raman probe for early cancer detection in the peripheral lung. J. Biophotonics 11:e201800055. doi: 10.1002/jbio.201800055

Meade, A. D., Clarke, C., Draux, F., Sockalingum, G. D., Manfait, M., Lyng, F. M., et al. (2010). Studies of chemical fixation effects in human cell lines using Raman microspectroscopy. Anal. Bioanal. Chem. 396, 1781-1791. doi: 10.1007/s00216-009-3411-7

Mo, J., Zheng, W., Low, J. J., Ng, J., Ilancheran, A., and Huang, Z. (2009). High wavenumber Raman spectroscopy for in vivo detection of cervical dysplasia. Anal Chem. 81, 8908-8915. doi: 10.1021/ac9015159

Movasaghi, Z., Rehman, S., and Rehman, I. U. (2007). Raman spectroscopy of biological tissues. Appl. Spectrosc. Rev. 42, 493-541. doi: 10.1080/05704920701551530

Nguyen, T. T., Gobinet, C., Feru, J., Pasco, S. B., Manfait, M., and Piot, O. (2012). Characterization of type I and IV collagens by Raman microspectroscopy: identification of spectral markers of the dermo-epidermal junction. Spectroscopy 27:7. doi: 10.1155/2012/6 86183

O’Brien, C. M., Herington, J. L., Brown, N., Pence, I. J., Paria, B. C., Slaughter, J. C., et al. (2017). In vivo Raman spectral analysis of impaired cervical remodeling in a mouse model of delayed parturition. Sci Rep. 7:6835. doi: 10.1038/s41598-017-07047-5

O’Connell, G. D., Lima, E. G., Bian, L., Chahine, N. O., Albro, M. B., Cook, J. L., et al. (2012). Toward engineering a biological joint replacement. J Knee Surg. 25, 187-196. doi: 10.1055/s-0032-1319783

Olejnik, C., Falgayrac, G., During, A., Vieillard, M. H., Maes, J. M., Cortet, B., et al. (2014). Molecular alterations of bone quality in sequesters of bisphosphonates-related osteonecrosis of the jaws. Osteoporosis Int. 25, 747-756. doi: 10.1007/s00198-013-2514-3

Oshima, Y., Sato, H., Kajiura-Kobayashi, H., Kimura, T., Naruse, K., and Nonaka, S. (2012). Light sheet-excited spontaneous Raman imaging of a living fish by optical sectioning in a wide field Raman microscope. Opt. Express. 20, 16195-16204. doi: 10.1364/OE.20.016195

Paidi, S. K., Rizwan, A., Zheng, C., Cheng, M., Glunde, K., and Barman, I. (2017). Label-free Raman spectroscopy detects stromal adaptations in premetastatic lungs primed by breast cancer. Cancer Res. 77, 247-256. doi: 10.1158/0008-5472.CAN-16-1862

Parekh, S. H., Lee, Y. J., Aamer, K. A., and Cicerone, M. T. (2010). Label-free cellular imaging by broadband coherent anti-Stokes Raman scattering microscopy. Biophys. J. 99, 2695-2704. doi: 10.1016/j.bpj.2010. 08.009

Patil, C. A., Kirshnamoorthi, H., Ellis, D. L., van Leeuwen, T. G., and MahadevanJansen, A. (2011). A clinical instrument for combined raman spectroscopyoptical coherence tomography of skin cancers. Lasers Surg. Med. 43, 143-151. doi: $10.1002 / \mathrm{lsm} .21041$

Pavlou, E., Zhang, X., Wang, J., and Kourkoumelis, N. (2018). Raman spectroscopy for the assessment of osteoarthritis. Ann. Joint. 2018:10. doi: 10.21037/aoj.2018.09.10

Pearle, A. D., Warren, R. F., and Rodeo, S. A. (2005). Basic science of articular cartilage and osteoarthritis. Clin. Sports Med. 24, 1-12. doi: 10.1016/j.csm.2004.08.007

Pence, I. J., Beaulieu, D. B., Horst, S. N., Bi, X., Herline, A. J., Schwartz, D. A., et al. (2017). Clinical characterization of in vivo inflammatory bowel disease with Raman spectroscopy. Biomed. Opt. Express. 8, 524-535. doi: 10.1364/BOE.8.000524

Pousty, I., Bari-Khan, M. A., and Butler, W. F. (1975). Leaching of glycosaminoglycans from tissues by the fixatives formalin-saline and formalincetrimide. Histochem. J. 7, 361-365. doi: 10.1007/BF01007021
Prabhu, S. D., and Frangogiannis, N. G. (2016). The biological basis for cardiac repair after myocardial infarction: from inflammation to fibrosis. Circ. Res. 119, 91-112. doi: 10.1161/CIRCRESAHA.116.303577

Puchtler, H., Meloan, S. N., and Waldrop, F. S. (1988). Are picro-dye reactions for collagens quantitative? Histochemistry 88, 243-256.

Puppels, G. J., de Mul, F. F. M., Otto, C., Greve, J., Robert-Nicoud, M., Arndt-Jovin, D. J., et al. (1990). Studying single living cells and chromosomes by confocal Raman microspectroscopy. Nature 347, 301-303. doi: 10.1038/347301a0

Querido, W., Falcon, J. M., Kandel, S., and Pleshko, N. (2017). Vibrational spectroscopy and imaging: applications for tissue engineering. Analyst 142, 4005-4017. doi: 10.1039/C7AN01055A

Raghavan, M., Sahar, N. D., Wilson, R. H., Mycek, M.-A., Pleshko, N., Kohn, D. H., et al. (2010). Quantitative polarized Raman spectroscopy in highly turbid bone tissue. J. Biomed. Opt. 15:037001. doi: 10.1117/1.3426310

Ryabchykov, O., Popp, J., and Bocklitz, T. (2018). Fusion of MALDI spectrometric imaging and raman spectroscopic data for the analysis of biological samples. Front. Chem. 6:257. doi: 10.3389/fchem.2018.00257

Rygula, A., Majzner, K., Marzec, K. M., Kaczor, A., Pilarczyk, M., and Baranska, M. (2013). Raman spectroscopy of proteins: a review. J. Raman Spectrosc. 44, 1061-1076. doi: 10.1002/jrs.4335

Santi, P. A., Aldaya, R., Brown, A., Johnson, S., Stromback, T., Cureoglu, S., et al. (2016). Scanning electron microscopic examination of the extracellular matrix in the decellularized mouse and human cochlea. J. Assoc. Res. Otolaryngol. 17, 159-171. doi: 10.1007/s10162-016-0562-Z

Schie, I. W., Krafft, C., and Popp, J. (2015). Applications of coherent Raman scattering microscopies to clinical and biological studies. Analyst 140, 3897-3909. doi: 10.1039/C5AN00178A

Schulmerich, M. V., Cole, J. H., Dooley, K. A., Morris, M. D., Kreider, J., Goldstein, S. A., et al. (2008b). Noninvasive Raman tomographic imaging of canine bone tissue. J. Biomed. Opt. 13, 1-3. doi: 10.1117/1.2904940

Schulmerich, M. V., Srinivasan, S., Kreider, J., Cole, J. H., Dooley, K. A., Goldstein, S. A., et al. (2008a). "Raman tomography of tissue phantoms and bone tissue," in Proceedings Volume 6853, Biomedical Optical Spectroscopy. San Jose, CA: SPIE.

Scott, J. E., and Stockwell, R. A. (1967). On the use and abuse of the critical electrolyte concentration approach to the localization of tissue polyanions. $J$. Histochem. Cytochem. 15, 111-113. doi: 10.1177/15.2.111

Shetty, G., Kendall, C., Shepherd, N., Stone, N., and Barr, H. (2006). Raman spectroscopy: elucidation of biochemical changes in carcinogenesis of oesophagus. Br. J. Cancer 94, 1460-1464. doi: 10.1038/sj.bjc.6603102

Shim, M. G., Song, L. M., Marcon, N. E., and Wilson, B. C. (2000). In vivo near-infrared Raman spectroscopy: demonstration of feasibility during clinical gastrointestinal endoscopy. Photochem. Photobiol. 72, 146-150. doi: 10.1562/0031-8655(2000)0720146IVNIRS2.0.CO2

Shoulders, M. D., and Raines, R. T. (2009). Collagen structure and stability. Ann. Rev. Biochem. 78, 929-958. doi: 10.1146/annurev.biochem.77.032207.120833

Shu, C., Chen, K., Lynch, M., Maher, J. R., Awad, H. A., and Berger, A. J. (2018). Spatially offset Raman spectroscopy for in vivo bone strength prediction. Biomed. Opt. Express. 9, 4781-4791. doi: 10.1364/BOE.9.004781

Silva, W. R., Graefe, C. T., and Frontiera, R. R. (2016). Toward Label-free super-resolution microscopy. ACS Photonics 3, 79-86. doi: 10.1021/acsphotonics.5b00467

Spalazzi, J. P., Boskey, A. L., Pleshko, N., and Lu, H. H. (2013). Quantitative mapping of matrix content and distribution across the ligament-to-bone insertion. PLoS ONE 8:e74349. doi: 10.1371/journal.pone.0074349

Stantchev, R. I., Mansfield, J. C., Edginton, R. S., Hobson, P., Palombo, F., and Hendry, E. (2018). Subwavelength hyperspectral THz studies of articular cartilage. Sci Rep. 8:6924. doi: 10.1038/s41598-018-25057-9

Stone, N., Kendall, C., Smith, J., Crow, P., and Barr, H. (2004). Raman spectroscopy for identification of epithelial cancers. Faraday Discussions 126, 141-57; discussion 69-83. doi: 10.1039/b304992b

Surmacki, J., Musial, J., Kordek, R., and Abramczyk, H. (2013). Raman imaging at biological interfaces: applications in breast cancer diagnosis. Mol. Cancer 12:48. doi: 10.1186/1476-4598-12-48

Takahashi, Y., Sugano, N., Takao, M., Sakai, T., Nishii, T., and Pezzotti, G. (2014). Raman spectroscopy investigation of load-assisted microstructural alterations in human knee cartilage: preliminary study into diagnostic potential for osteoarthritis. J. Mech. Behav. Biomed. Mater. 31, 77-85. doi: 10.1016/j.jmbbm.2013.02.014 
Teh, S. K., Zheng, W., Ho, K. Y., Teh, M., Yeoh, K. G., and Huang, Z. (2008). Diagnosis of gastric cancer using near-infrared Raman spectroscopy and classification and regression tree techniques. J. Biomed. Opt. 13:034013. doi: $10.1117 / 1.2939406$

Tfayli, A., Gobinet, C., Vrabie, V., Huez, R., Manfait, M., and Piot, O. (2009). Digital dewaxing of Raman signals: discrimination between nevi and melanoma spectra obtained from paraffin-embedded skin biopsies. Appl. Spectrosc. 63, 564-570. doi: 10.1366/000370209788347048

Tong, L., Hao, Z., Wan, C., and Wen, S. (2018). Detection of depth-depend changes in porcine cartilage after wear test using Raman spectroscopy. J. Biophotonics 11:e201700217. doi: 10.1002/jbio.201700217

Unal, M., and Akkus, O. (2015). Raman spectral classification of mineraland collagen-bound water's associations to elastic and post-yield mechanical properties of cortical bone. Bone 81, 315-326. doi: 10.1016/j.bone.2015.07.024

Unal, M., Akkus, O., Sun, J., Cai, L., Erol, U. L., Sabri, L., et al. (2019). Raman spectroscopy-based water content is a negative predictor of articular human cartilage mechanical function. Osteoarthritis Cartilage 27, 304-313. doi: $10.1016 /$ j.joca.2018.10.003

Utzinger, U., and Richards-Kortum, R. R. (2003). Fiber optic probes for biomedical optical spectroscopy. J. Biomed. Opt. 8, 121-147. doi: 10.1117/1.1528207

Votteler, M., Carvajal Berrio, D. A., Pudlas, M., Walles, H., Stock, U. A., and Schenke-Layland, K. (2012). Raman spectroscopy for the non-contact and nondestructive monitoring of collagen damage within tissues. J. Biophotonics 5, 47-56. doi: 10.1002/jbio.201100068

White, E. S. (2015). Lung extracellular matrix and fibroblast function. Ann. Am. Thoracic Soc. 12, S30-S33. doi: 10.1513/AnnalsATS.201406-240MG
Widjaja, E., Zheng, W., and Huang, Z. (2008). Classification of colonic tissues using near-infrared Raman spectroscopy and support vector machines. Int. J. Oncol. 32, 653-662. doi: 10.3892/ijo.32.3.653

Wilson, N. H., and Gardner, D. L. (1980). Influence of aqueous fixation on articular surface morphology. A reflected light interference microscope study. J. Pathol. 131, 333-338. doi: 10.1002/path.17113 10405

You, A. Y. F., Bergholt, M. S., St-Pierre, J. P., Kit-Anan, W., Pence, I. J., Chester, A. H., et al. (2017). Raman spectroscopy imaging reveals interplay between atherosclerosis and medial calcification in the human aorta. Sci. Adv. 3:e1701156. doi: 10.1126/sciadv.1701156

Zhao, J., Lui, H., McLean, D. I., and Zeng, H. (2007). Automated autofluorescence background subtraction algorithm for biomedical Raman spectroscopy. Appl. Spectrosc. 61, 1225-1232. doi: 10.1366/0003702077825 97003

Conflict of Interest: The authors declare that the research was conducted in the absence of any commercial or financial relationships that could be construed as a potential conflict of interest.

Copyright (C) 2019 Bergholt, Serio and Albro. This is an open-access article distributed under the terms of the Creative Commons Attribution License (CC BY). The use, distribution or reproduction in other forums is permitted, provided the original author(s) and the copyright owner(s) are credited and that the original publication in this journal is cited, in accordance with accepted academic practice. No use, distribution or reproduction is permitted which does not comply with these terms. 